\title{
RATIONAL POINTS ON LOG FANO THREEFOLDS OVER A FINITE FIELD
}

\author{
YOSHINORI GONGYO, YUSUKE NAKAMURA, AND HIROMU TANAKA
}

\begin{abstract}
We prove the $W \mathcal{O}$-rationality of klt threefolds and the rational chain connectedness of klt Fano threefolds over a perfect field of characteristic $p>5$. As a consequence, any klt Fano threefold over a finite field has a rational point.
\end{abstract}

\section{Contents}

1. Introduction

2. Preliminaries

2.1. Notation and convention

2.2. Log pairs

2.3. MMP over a perfect field

2.4. Special fibers of log Fano contractions

2.5. Witt vectors

2.6. Fundamental properties of Witt vector cohomologies

3. $W \mathcal{O}$-rationality of klt threefolds

3.1. WO-rationality under pl-contractions and pl-flips

3.2. Normalization for plt centers under pl-contractions and pl-flips

3.3. $W \mathcal{O}$-rationality of klt threefolds

3.4. Examples

4. Rational chain connectedness

4.1. Birational and relative cases

4.2. Global case

5. Rational points on log Fano contractions over finite fields

References

\section{INTRODUCTION}

The problem whether a certain system of polynomials has a common solution or not is classical but has been of great interest in the history of mathematics. In the geometrical context, Fano varieties defined over a certain field $k$ are believed to have a $k$-rational point. For instance, the classically known Chevalley-Warning theorem states that homogeneous polynomials $f_{1}, \ldots, f_{l} \in \mathbb{F}_{q}\left[x_{0}, \ldots, x_{n}\right]$ with $(n+1)$-many variables over a finite field $\mathbb{F}_{q}$ have a non-trivial common solution if $\sum_{1 \leq i \leq l} \operatorname{deg} f_{i} \leq n$ holds. More

2010 Mathematics Subject Classification. Primary 14J45; Secondary 14G05, 14E30.

Key words and phrases. rational points, Fano varieties, Witt rationality, rational chain connectedness. 
precisely, it states that the number of common solution is divisible by the characteristic $p$ of the field $\mathbb{F}_{q}$. Geometrically, it can be interpreted as that the number of the $\mathbb{F}_{q}$-rational points on a complete intersection Fano variety is one modulo $p$. This kind of problem was answered in the smooth case by Esnault [Esn03]:

Theorem 1.1 (Esnault [Esn03]). Let $X$ be a smooth geometrically connected projective variety over a finite field $\mathbb{F}_{q}$ with $q$ elements. If $-K_{X}$ is ample, then the number of $\mathbb{F}_{q}$-rational points satisfies $\# X\left(\mathbb{F}_{q}\right) \equiv 1(\bmod q)$.

One motivation of this paper is to generalize this result to three dimensional varieties with singularities. Indeed we prove that the same statement holds for three dimensional varieties of Fano type defined over a finite field of characteristic larger than five.

Theorem 1.2. Let $X$ be a three dimensional normal geometrically connected projective variety over a finite field $\mathbb{F}_{q}$ of characteristic larger than five. If $X$ is of Fano type, then the number of $\mathbb{F}_{q}$-rational points satisfies $\# X\left(\mathbb{F}_{q}\right) \equiv 1$ $(\bmod q)$.

Here, we say that a normal projective variety $X$ defined over a field is of Fano type if there exists an effective $\mathbb{Q}$-divisor $\Delta$ such that $(X, \Delta)$ is klt (see Subsection 2.2 for the definition) and $-\left(K_{X}+\Delta\right)$ is ample.

By [BBE07, Theorem 1.1] and the Lefschetz trace formula, Theorem 1.2 is a consequence of the following proposition, which states the vanishing of the Witt vector cohomology (see Subsection 2.5 for the definition).

Theorem 1.3. Let $X$ be a three dimensional normal projective variety over a perfect field of characteristic larger than five. If $X$ is of Fano type, then $H^{i}\left(X, W \mathcal{O}_{X, \mathbb{Q}}\right)=0$ holds for $i>0$.

It can be seen as an analogy of the vanishing of the structure sheaf $H^{i}\left(X, \mathcal{O}_{X}\right)=$ $0(i>0)$, which is true in characteristic zero for varieties of Fano type of arbitrary dimension by the Kawamata-Viehweg vanishing theorem. In the case of positive characteristic, we do not know whether the vanishing $H^{i}\left(X, \mathcal{O}_{X}\right)=0(i>0)$ holds or not by the lack of the Kodaira vanishing theorem, although the vanishing $H^{i}\left(X, \mathcal{O}_{X}\right)=0(i>0)$ implies the vanishing of $H^{i}\left(X, W \mathcal{O}_{X, \mathbb{Q}}\right)=0(i>0)$ (cf. Lemma 2.19). However, by BE08, the vanishing in Theorem 1.3 is known to be true for a variety $X$ with the following two properties:

- $X$ has $W \mathcal{O}$-rational singularities, and

- $X$ is rationally chain connected.

Hence, this paper is devoted to prove the following two theorems.

Theorem 1.4 (= Theorem 3.16). Let $(X, \Delta)$ be a three dimensional klt pair over a perfect field in characteristic larger than five. Then $X$ has $W \mathcal{O}$ rational singularities, that is, there exists a proper birational morphism $\varphi$ : $V \rightarrow X$ from a smooth threefold $V$ such that $R^{i} \varphi_{*}\left(W \mathcal{O}_{V, \mathbb{Q}}\right)=0$ holds for $i>0$.

Theorem 1.5 (= Proposition4.11). Let $X$ be a three dimensional projective variety of Fano type over a perfect field of characteristic larger than five. Then $X$ is rationally chain connected. 
Remark 1.6. In Section 5 , we shall prove Theorem 1.2 directly from Theorem 1.4 and Theorem 1.5 using the argument in [Esn03] and [BBE07].

Theorem 1.4 can be seen as an analogy of the following fact in characteristic zero which is proved in [KMM87, Section 1-3] (cf. [KM98, Theorem $5.2])$ :

- klt varieties have rational singularities, that is, there exists a proper birational morphism $\varphi: V \rightarrow X$ from a smooth variety $V$ such that $R^{i} \varphi_{*}\left(\mathcal{O}_{V}\right)=0$ holds for $i>0$.

It is known to fail in positive characteristic. In Subsection 3.4, we shall see examples in characteristic two which are klt but with non-rational singularities. This example (Proposition 3.19) is four-dimensional. However, in dimension three, we do not know whether klt singularities are always rational singularities or not.

On the other hand, related to Theorem 1.5, Campana and Kollár-MiyaokaMori showed in the 1990's that smooth Fano varieties over characteristic zero are rationally connected KMM92, Cam92. Recently, Zhang and Hacon$\mathrm{M}^{\mathrm{c}}$ Kernan generalized this to log Fano varieties over characteristic zero [Zha06, HM07]. Then, it is natural to ask the following question: are smooth Fano varieties over a positive characteristic field rationally connected? This is still an open problem, but it is known that they are rationally chain connected (cf. [Kol96, Ch V, 2.14]). The first and third authors proved the above theorem for globally $F$-regular threefolds with Z. Li, Z. Patakfalvi, K. Schwede and R. Zong in [GLP ${ }^{+}$15] (see [SS10, Definition 3.1] for the definition of globally $F$-regular varieties). In this paper, we generalize this result to varieties of Fano type by the similar strategy to $\left[\mathrm{GLP}^{+} 15\right]$. After finishing the work [GLP ${ }^{+}$15], we noticed that Shokurov in Sho00] claimed that this problem can be reduced to the standard conjecture in the minimal model theory and the resolution of singularities. His argument looks similar to ours but we could not get the proof in positive characteristic. Hence, also for the reader's convenience, we include the proof of the rationally chain connectedness.

The paper is organized as follows: in Section 2, we review some results on the minimal model theory in positive characteristic. Some results are generalized over a perfect field in order to apply them to finite fields in the latter sections. Further in Subsection 2.5 and 2.6, we review the definition of the Witt vector cohomology and study its properties. In Section 3 , we prove the $W \mathcal{O}$-rationality of klt threefolds in characteristic $p>5$ (Theorem 1.4). Further, as a consequence in characteristic $p \leq 5$, we reduce the problem on the existence of flips to a certain special case (Theorem 3.15). It suggests that the Witt vector cohomology is helpful also for the minimal model theory itself. In Section 4, we prove the rational chain connectedness of threefolds of Fano type in characteristic $p>5$ (Theorem 1.5). We also treat the relative setting (Theorem 4.1). In Section 5, we prove the main theorem (Theorem 1.2) and the vanishing theorem (Theorem 1.3).

After we put the first draft of this paper on arXiv, Wang also put a preprint Wan there, which is on the rational chain connectedness. We note here that Theorem A and B in Wan] follow from Theorem 4.1] in our paper. 
Acknowledgements. We would like to thank Professors Yujiro Kawamata and Shunsuke Takagi for warm encouragements and giving advices. We would also like to thank Professors Paolo Cascini and Hélène Esnault for the discussions. The first author also wish to thank Professors Shigefumi Mori and Caucher Birkar for discussing the rationality of the bases of conic bundles and the rational chain connectedness of conic bundles. The first and third authors wish to express their deep gratitude to the collaborators of $\mathrm{GLP}^{+}$15]: Zhiyuan Li, Zsolt Patakfalvi, Karl Schwede and Runhong Zong for many discussions during working our collaboration. We also appreciate Professors Jean-Louis Colliot-Thélène and János Kollár for discussing the definition of the rational chain connectedness. We appreciate the referees for careful reading this paper and pointing out some mistakes. In particular, one of the referees pointed out the difference of the definition of Witt-rationality between the one in CR12 and the one in the previous version of this paper (cf Remark 2.17).

The first author is partially supported by the Grant-in-Aid for Scientific Research (KAKENHI No. 26707002). The second author is partially supported by the Grant-in-Aid for Scientific Research (KAKENHI No. 25-3003).

\section{Preliminaries}

2.1. Notation and convention. We summarize notation of this paper.

- Throughout this paper, we work over a perfect field $k$ of characteristic $p>0$ unless otherwise specified.

- We often identify an invertible sheaf $L$ with the divisor corresponding to $L$. For example, we use the additive notation $L+A$ for a line bundle $L$ and a divisor $A$.

- For a coherent sheaf $F$ and a Cartier divisor $L$, we define $F(L):=$ $F \otimes \mathcal{O}_{X}(L)$.

- We say that $X$ is a variety over $k$ if $X$ is an integral scheme which is separated and of finite type over $k$. We say that $X$ is a curve (resp. a surface, a threefold) if $X$ is a variety over $k$ with $\operatorname{dim} X=1$ (resp. $\operatorname{dim} X=2, \operatorname{dim} X=3)$.

2.2. Log pairs. We recall some notation in the theory of singularities in the minimal model program. For more details, we refer the reader KM98, Section 2.3] and [Kol13.

We say that $(X, \Delta)$ is a $\log$ pair if $X$ is a normal variety and $\Delta$ is an effective $\mathbb{Q}$-divisor such that $K_{X}+\Delta$ is $\mathbb{Q}$-Cartier. For a proper birational morphism $f: X^{\prime} \rightarrow X$ from a normal variety $X^{\prime}$ and a prime divisor $E$ on $X^{\prime}$, the discrepancy of $(X, \Delta)$ at $E$ is defined as

$$
a_{E}(X, \Delta):=\operatorname{coeff}_{E}\left(K_{X^{\prime}}-f^{*}\left(K_{X}+\Delta\right)\right) .
$$

We say that a $\log$ pair $(X, \Delta)$ is $\log$ canonical if $a_{E}(X, \Delta) \geq-1$ for any divisor $E$ over $X$. Further, we say that a $\log$ pair $(X, \Delta)$ is Kawamata log terminal, $k l t$ for short (resp. purely log terminal, plt for short), if $a_{E}(X, \Delta)>$ -1 for any divisor (resp. exceptional divisor) $E$ over $X$.

We call a $\log$ pair $(X, \Delta)$ divisorial log terminal (dlt for short) when there exists a $\log$ resolution $f: Y \rightarrow X$ such that $a_{E}(X, \Delta)>-1$ for any $f$-exceptional divisor $E$ on $Y$. 
We recall here the definition of $\log$ resolution. Let $D$ be a closed subset of a smooth scheme $X$ (over a perfect field $k$ ) and let $D_{1}, \ldots, D_{n}$ be the irreducible components of $D$ with the reduced scheme structures. We say that $D$ is simple normal crossing if the scheme-theoretic intersection $\bigcap_{j \in J} D_{j}$ is smooth over $k$ for every subset $J \subset\{1, \ldots, n\}$ (cf. [KEW, Subsection 2.1]). For a variety $X$ and a closed subset $Z$ of $X$, we say that $f: Y \rightarrow X$ is a $\log$ resolution of $(X, Z)$ when $f$ is a proper birational morphism from a smooth variety $Y$ such that $f^{-1}(Z)$ is purely codimension one and $\operatorname{Ex}(f) \cup f^{-1}(Z)$ is simple normal crossing. In dimension three, there exists a log resolution for such a pair $(X, Z)$ by [CP08] and Abh66.

Remark 2.1. In Lemma 3.6 we will need to work on a more general setting than the one above. In this setting, $X$ is allowed to be a normal, excellent scheme over a possibly imperfect field. All the above definitions can be extended to this setting. For details, in particular for the precise definitions of $K_{X}$, we refer to [Kol13, Definition 2.4, Definition 2.8].

2.3. MMP over a perfect field. For later use, we summarize some results on the minimal model theory. First we collect some results which work in an arbitrary positive characteristic.

Lemma 2.2 (Relative pseudo-effective cone theorem). Let $k$ be a perfect field of characteristic $p>0$. Let $X$ be a $\mathbb{Q}$-factorial threefold which is projective over a separated scheme $U$ of finite type over $k$. Let $\Delta$ be a $\mathbb{Q}$ divisor on $X$ whose coefficients are contained in $[0,1]$. Assume that $K_{X}+\Delta$ is pseudo-effective over $U$. Then, for every ample $\mathbb{Q}$-divisor $A$, there exists a finitely many curves $C_{1}, \cdots, C_{r}$ such that

$$
\overline{N E}(X / U)=\overline{N E}(X / U)_{K_{X}+\Delta+A \geq 0}+\sum_{i=1}^{r} \mathbb{R}_{\geq 0}\left[C_{i}\right] .
$$

Proof. We can perturb $\Delta$, by using an ample $\mathbb{Q}$-divisor $A$, and we may assume that

$$
K_{X}+\Delta \sim_{\mathbb{Q}, U} E
$$

where $E$ is an effective $\mathbb{Q}$-divisor. Then, the assertion is proved by the same proof as in [Kee99, Proposition 0.6] (cf. [Tana, Theorem 6.6]).

Lemma 2.3 (Relative Keel's theorem). Let $k$ be a perfect field of characteristic $p>0$. Let $\pi: X \rightarrow U$ be a projective morphism from a normal variety $X$ to a separated scheme $U$ of finite type over $k$. Let $L$ be a $\pi$-nef $\mathbb{Q}$-Cartier $\mathbb{Q}$-divisor on $X$. Assume that $L=A+E$ where $A$ is a $\pi$-ample $\mathbb{Q}$-Cartier $\mathbb{Q}$-divisor and $E$ is an effective $\mathbb{Q}$-divisor on $X$. If $\left.L\right|_{\operatorname{Supp} E}$ is $\pi$-semi-ample, then $L$ is $\pi$-semi-ample.

Proof. It is proved by the same proof as in [CMM14, Lemma 3.3] (cf. [Xu15, Proposition 2.7]).

Lemma 2.4 (Pl-contraction theorem). Let $k$ be a perfect field of characteristic $p>0$. Let $(X, \Delta)$ be a $\mathbb{Q}$-factorial dlt threefold with a projective morphism $\pi: X \rightarrow U$ to a separated scheme $U$ of finite type over $k$. Let $L$ be a $\pi$-nef and $\pi$-big Cartier divisor on $X$ such that $\overline{N E}(X / U) \cap L^{\perp}=: R$ is a $\left(K_{X}+\Delta\right)$-negative extremal ray of $\overline{N E}(X / U)$. Assume the following two conditions. 
- $S \cdot R<0$ for some irreducible component $S$ of $\llcorner\Delta\lrcorner$.

- The normalization of $S$ is a universal homeomorphism.

Then, $L$ is $\pi$-semi-ample.

Proof. We may assume that $(X, \Delta)$ is plt with $S=\llcorner\Delta\lrcorner$. By the assumption, $A:=L-\epsilon S$ is $\pi$-ample for small $\epsilon>0$. Since $L=A+\epsilon S$, it suffices to show that $\left.L\right|_{S}$ is $\pi$-semi-ample by Lemma 2.3. Since the normalization $S^{N} \rightarrow S$ is a universal homeomorphism, it is enough to prove that $\left.L\right|_{S^{N}}$ is $\pi$-semi-ample (cf. [Kee99, Lemma 1.4]). By adjunction, the new pair $\left(S^{N}, \Delta_{S^{N}}\right)$, defined by

$$
\left.\left(K_{X}+\Delta\right)\right|_{S^{N}}=K_{S^{N}}+\Delta_{S^{N}}
$$

is klt, where $\Delta_{S^{N}}=\operatorname{Diff}_{S^{N}}(\Delta)$ is the different in the sense of Kol13, Section 4.1]. Since

$$
\left.m L\right|_{S^{N}}-\left(K_{S^{N}}+\Delta_{S^{N}}\right)=\left.\left(m L-\left(K_{X}+\Delta\right)\right)\right|_{S^{N}}
$$

is $\pi$-ample for $m \gg 0$ by the assumption, it follows that $\left.L\right|_{S^{N}}$ is $\pi$-semiample by [Tan14, Theorem 6.9].

Lemma 2.5 (R1 for plt centers). Let $k$ be a field. Let $(X, S+B)$ be a plt pair over $k$ such that $\llcorner S+B\lrcorner=S$. Then, $S$ is regular in codimension one, that is, there exists an open subset $U \subset S$ such that $\operatorname{codim}_{S}(S \backslash U) \geq 2$ and that $U$ is regular.

Proof. Set $\nu: S^{N} \rightarrow S$ to be the normalization. Then the log pair $\left(S_{N}, \Delta_{S^{N}}\right)$, defined by $\left.\left(K_{X}+S+B\right)\right|_{S^{N}}=K_{S^{N}}+\Delta_{S^{N}}$, is klt, where $\Delta_{S^{N}}=\operatorname{Difff}_{S^{N}}(\Delta)$ is the different in the sense of [Kol13, Section 4.1]. Then $\left\llcorner\Delta_{S^{N}}\right\lrcorner=0$. If $S$ is not regular in codimension one, then the corresponding divisor to the codimension one part of the conductor ideal appears in $\left\llcorner\Delta_{S^{N}}\right\lrcorner$ by [Kol13, Proposition 4.5 (1)]. This contradicts $\left\llcorner\Delta_{S^{N}}\right\lrcorner=0$.

Theorem 2.6 (Special termination). Let $k$ be a perfect field and $Z$ a quasiprojective variety over $k$. Let $(X, S+B)$ be a $\mathbb{Q}$-factorial dlt threefold, projective over $Z$, such that $\llcorner S+B\lrcorner=S$. Then every sequence of $\left(K_{X}+\right.$ $S+B)$-flips over $Z$ terminates around $S$.

Proof. The arguments of the proof in Fuj07, Theorem 4.2.1] work by replacing $S_{i}$ with the normalization $S_{i}^{\nu}$ of $S_{i}$.

Second, we summarize results in characteristic $p>5$. Almost all the results are known for the case where $k$ is algebraically closed. We shall run an MMP over a finite field in Section 5, hence we need to generalize them over perfect fields.

Remark 2.7. We summarize here what kind of properties are preserved under the base change to the algebraic closure.

(1) Let $X$ be a normal variety over a perfect field. Then its base change $X^{\prime}:=X \times_{k} \bar{k}$ to the algebraic closure $\bar{k}$ is also normal. In particular, each connected component of $X^{\prime}$ is reduced and irreducible. Further, if a pair $(X, \Delta)$ is log canonical (resp. klt, plt, dlt), then so is $\left(X^{\prime}, \Delta^{\prime}:=\Delta \times_{k} \bar{k}\right.$ ) (see Proposition 2.15 in [Kol13] and Warning below it). However, the $\mathbb{Q}$-factoriality is not preserved in general. 
Let $L$ be a line bundle on $X$ and let $L^{\prime}$ be its pull-back to $X^{\prime}$. Then, $L$ is ample (resp. nef, big, semi-ample) if and only if so is $L^{\prime}$.

(2) Let $k \subseteq k^{\prime}$ be a field extension and $\pi: X \rightarrow S$ a morphism of schemes over $k$, and let $L$ be a line bundle on $X$. Denote the base change $X \times_{k} k^{\prime} \rightarrow S \times \times_{k} k^{\prime}$ by $\pi^{\prime}: X^{\prime} \rightarrow S^{\prime}$ and denote the pull-back $L \otimes_{\mathcal{O}_{X}} \mathcal{O}_{X^{\prime}}$ by $L^{\prime}$. Then the following hold:

(a) $L$ is base point free over $S$ if and only if so is $L^{\prime}$ over $S^{\prime}$, and

(b) $\bigoplus_{l \geq 0} \pi_{*}\left(L^{\otimes l}\right)$ is a finitely generated algebra over $\mathcal{O}_{S}$ if and only if so is $\bigoplus_{l \geq 0} \pi_{*}^{\prime}\left(L^{\prime \otimes l}\right)$ over $\mathcal{O}_{S^{\prime}}$ (Gro65, Proposition 2.7.1]).

Indeed, we have $\pi_{*}\left(L^{\otimes l}\right) \otimes_{\mathcal{O}_{X}} \mathcal{O}_{X^{\prime}} \cong \pi_{*}^{\prime}\left(L^{\prime \otimes l}\right)$ by the flat base change under Spec $k^{\prime} \rightarrow$ Spec $k$.

Lemma 2.8. Let $(X, \Delta)$ be a klt pair over a perfect field with $\operatorname{dim} X \leq 3$. Let $A$ be a nef and big $\mathbb{Q}$-Cartier $\mathbb{Q}$-divisor. Then there exists an effective $\mathbb{Q}$-divisor $A^{\prime} \sim_{\mathbb{Q}} A$ such that $\left(X, \Delta+A^{\prime}\right)$ is klt.

Proof. By the existence of a $\log$ resolution of $(X, \Delta)$, we may assume that $X$ is smooth and quasi-projective, and that $\Delta$ is simple normal crossing, and $A$ is ample. If $k$ is infinite, then we can apply the usual Bertini theorem. If $k$ is a finite field, we can apply the same argument as in [Poo04, Theorem 1.1 or 1.2].

Theorem 2.9 (Relative base point free theorem). Let $k$ be a perfect field of characteristic $p>5$. Let $(X, \Delta)$ be a klt threefold over $k$, equipped with a projective morphism $f: X \rightarrow Z$ to a quasi-projective variety $Z$ over $k$ such that $f_{*} \mathcal{O}_{X}=\mathcal{O}_{Z}$. If $L$ is a $f$-nef Cartier divisor such that $L-\left(K_{X}+\Delta\right)$ is $f$-nef and $f$-big, then $L$ is $f$-semi-ample.

Proof. By Remark 2.7, we may assume that $k$ is algebraically closed. When $Z$ is projective, the assertion follows from [BW, Theorem 1.2].

For the general case, we may assume that $X$ is $\mathbb{Q}$-factorial by Bir, Theorem 1.6]. Let $A$ be an $f$-ample effective $\mathbb{Q}$-divisor such that

$$
A \sim_{f, \mathbb{Q}} L-\left(K_{X}+\Delta\right)
$$

and $(X, \Delta+A)$ is klt. We take a compactification $\bar{f}: \bar{X} \rightarrow \bar{Z}$ of $f: X \rightarrow Z$ such that $\bar{X}$ and $\bar{Z}$ are projective normal threefolds. In particular, $\bar{f}_{*} \mathcal{O}_{\bar{X}}=$ $\mathcal{O}_{\bar{Z}}$. Let $\varphi: \widetilde{X} \rightarrow \bar{X}$ be a $\log$ resolution of $(\bar{X} \backslash X) \cup \operatorname{Supp}(\Delta+A)$ and we denote the composition by

$$
\widetilde{f}: \widetilde{X} \stackrel{\varphi}{\rightarrow} \bar{X} \stackrel{\bar{f}}{\rightarrow} \bar{Z}
$$

Let $\widetilde{\Delta}$ and $\widetilde{A}$ be the strict transforms on $\widetilde{X}$ of the closures of $\Delta$ and $A$, respectively. We set $E:=(1-\epsilon) \sum E_{i}$, where $\sum E_{i}$ is the sum of all the $\varphi$-exceptional prime divisors and $\epsilon$ is a sufficiently small rational number. Run a $\left(K_{\tilde{X}}+\widetilde{\Delta}+\widetilde{A}+E\right)$-MMP over $\bar{X}$ ([BW, Theorem 1.6]). Then the end result $\varphi^{\prime}:\left(\widetilde{X}^{\prime}, \widetilde{\Delta}^{\prime}+\widetilde{A}^{\prime}+E^{\prime}\right) \rightarrow \bar{X}$ is isomorphic over $X$ (hence $E^{\prime}$ is supported on $\left.\tilde{X}^{\prime} \backslash\left(\varphi^{\prime}\right)^{-1}(X)\right)$ since $X$ is $\mathbb{Q}$-factorial klt and $\epsilon$ is sufficiently small. Next run a $\left(K_{\widetilde{X}^{\prime}}+\widetilde{\Delta}^{\prime}+\widetilde{A}^{\prime}+E^{\prime}\right)$-MMP over $\bar{Z}$ ([BW, Theorem 1.6]). Since $K_{\widetilde{X}^{\prime}}+\widetilde{\Delta}^{\prime}+\widetilde{A}^{\prime}+E^{\prime}$ is already nef over $Z$ by the assumption, the resulting model $f^{\prime \prime}:\left(\widetilde{X}^{\prime \prime}, \widetilde{\Delta}^{\prime \prime}+\widetilde{A}^{\prime \prime}+E^{\prime \prime}\right) \rightarrow \bar{Z}$ is isomorphic to $f^{\prime}:\left(\widetilde{X}^{\prime}, \widetilde{\Delta}^{\prime}+\widetilde{A}^{\prime}+E^{\prime}\right) \rightarrow$ 
$\bar{Z}$ over $Z$. In particular, $f^{\prime \prime}:\left(\widetilde{X}^{\prime \prime}, \widetilde{\Delta}^{\prime \prime}+\widetilde{A}^{\prime \prime}+E^{\prime \prime}\right) \rightarrow \bar{Z}$ is a compactification of $f:(X, \Delta+A) \rightarrow Z$. Further, it follows that the $\mathbb{Q}$-divisor $K_{\widetilde{X}^{\prime \prime}}+\widetilde{\Delta}^{\prime \prime}+\widetilde{A}^{\prime \prime}+E^{\prime \prime}$ is $f^{\prime \prime}$-nef.

Thus, in order to show the $f$-semi-ampleness of $K_{X}+\Delta+A$, it is enough to show the $f^{\prime \prime}$-semi-ampleness of $K_{\widetilde{X}^{\prime \prime}}+\widetilde{\Delta}^{\prime \prime}+\widetilde{A}^{\prime \prime}+E^{\prime \prime}$ (since $E^{\prime \prime}$ is supported on $\left.\widetilde{X}^{\prime \prime} \backslash\left(f^{\prime \prime}\right)^{-1}(Z)\right)$. Since $\widetilde{A}^{\prime \prime}$ is $f^{\prime \prime}$-big, there exist an $f^{\prime \prime}$-ample $\mathbb{Q}$-divisor $M$ and an effective $\mathbb{Q}$-divisor $\Gamma$ such that $\widetilde{A}^{\prime \prime} \sim_{f^{\prime \prime}, \mathbb{Q}} M+\Gamma$. Take a rational number $\delta>0$ such that $\left(\widetilde{X}^{\prime \prime}, \widetilde{\Delta}^{\prime \prime}+(1-\delta) \widetilde{A}^{\prime \prime}+E^{\prime \prime}+\delta \Gamma\right)$ is klt. Then the $f^{\prime \prime}$-semi-ampleness of

$$
K_{\widetilde{X}^{\prime \prime}}+\widetilde{\Delta}^{\prime \prime}+\widetilde{A}^{\prime \prime}+E^{\prime \prime} \sim_{f^{\prime \prime}, \mathbb{Q}} K_{\widetilde{X}^{\prime \prime}}+\widetilde{\Delta}^{\prime \prime}+(1-\delta) \widetilde{A}^{\prime \prime}+E^{\prime \prime}+\delta \Gamma+\delta M
$$

follows from the projective case ([BW, Theorem 1.2]).

Theorem 2.10 (Existence of flips). Let $k$ be a perfect field of characteristic $p>5$. Let $f:(X, \Delta) \rightarrow Z$ be a flipping contraction from a $\mathbb{Q}$-factorial dlt threefold $(X, \Delta)$. Then a flip of $f$ exists.

Proof. We may assume that $(X, \Delta)$ is klt. The assertion follows from Bir, Theorem 1.3] and Remark 2.7 (2).

In dimension three and in characteristic $p>5$, the normality of plt centers is known (cf. Lemma 2.5).

Theorem 2.11 (Normality of plt centers). Let $k$ be a perfect field of characteristic $p>5$. Let $(X, \Delta)$ be a $\mathbb{Q}$-factorial dlt threefold over $k$. Then every irreducible component of $\llcorner\Delta\lrcorner$ is normal.

Proof. We may assume that $\Delta$ is a prime divisor, in particular the pair is plt. Then, the base change $\left(X \times_{k} \bar{k}, \Delta \times_{k} \bar{k}\right)$ to the algebraic closure is also plt. Since $\Delta \times_{k} \bar{k}$ is normal by Theorem 3.1 and Proposition 4.1 in [HX15], so is $\Delta$.

Theorem 2.12 (MMP with scaling). Let $k$ be a perfect field of characteristic $p>5$, and let $(X, \Delta)$ be a projective $\mathbb{Q}$-factorial klt threefold over $k$, equipped with a morphism $f: X \rightarrow Z$ to a projective variety $Z$ over $k$ with $f_{*} \mathcal{O}_{X}=$ $\mathcal{O}_{Z}$. Let $C$ be an effective $\mathbb{Q}$-divisor on $X$ which satisfies the following properties:

(1) $(X, \Delta+C)$ is klt,

(2) $C$ is $f$-big, and

(3) $K_{X}+\Delta+C$ is f-nef.

Then, we can run a $\left(K_{X}+\Delta\right)$-MMP over $Z$ with scaling of $C$ and it terminates.

Proof. By Theorem 2.9 and Theorem 2.10, the only problem to show is the termination of flips.

First, we show the assertion under the assumption that $k$ is algebraically closed and that $Z=$ Spec $k$. If $K_{X}+\Delta$ is not pseudo-effective, then the assertion follows from [BW, Theorem 1.5] because in this case, a $\left(K_{X}+\Delta\right)$ MMP with scaling of $C$ can be considered as a $\left(K_{X}+\Delta+\epsilon C\right)$-MMP with scaling of $C$ for some $\epsilon>0$. If $K_{X}+\Delta$ is pseudo-effective, then we can apply the same argument as in [BW, Proposition 4.4]. 
Second, we work on the case where $k$ is algebraically closed field but we do not impose any additional assumption on $Z$. Set $C^{\prime}=C+f^{*} H$ for $H$ a sufficiently ample divisor on $Z$. Then $C^{\prime}=C+f^{*} H$ is globally big and $K_{X}+\Delta+C^{\prime}$ is globally nef by the cone theorem. Moreover, by [Tanb, Theorem 1], $\left(X, \Delta^{\prime}\right)$ becomes klt again for some effective $\mathbb{Q}$-divisor $\Delta^{\prime}$ such that $\Delta^{\prime} \sim_{\mathbb{Q}} \Delta+f^{*} H$. Since $H$ is sufficiently ample, any $\left(K_{X}+\Delta\right)$ MMP over $Z$ with scaling of $C$ can be considered as a $\left(K_{X}+\Delta^{\prime}\right)$-MMP over Spec $k$ with scaling of $C^{\prime}$. Then the termination follows from the case where $Z=$ Spec $k$.

Third, let us go back to the general case, where $k$ is perfect. Let

$$
(X, \Delta)=\left(X_{1}, \Delta_{1}\right) \rightarrow\left(X_{2}, \Delta_{2}\right) \rightarrow \cdots
$$

be a $\left(K_{X}+\Delta\right)$-MMP over $Z$ with scaling of $C$. Suppose that this sequence consists of flips. Take the base change to the algebraic closure. The new log pairs after the base change might be non- $\mathbb{Q}$-factorial. However, by Bir12, Remark 2.9], taking a small $\mathbb{Q}$-factorialization for each log pair, we can construct a sequence of an MMP with scaling of some $C^{\prime}$ which satisfies the same properties as in (1)-(3). More precisely, There exists a small $\mathbb{Q}$ factorialization $\left(X_{i}^{\prime}, \Delta_{i}^{\prime}\right)$ such that the new sequence

$$
\left(X^{\prime}, \Delta^{\prime}\right)=\left(X_{1}^{\prime}, \Delta_{1}^{\prime}\right) \rightarrow\left(X_{2}^{\prime}, \Delta_{2}^{\prime}\right) \rightarrow \cdots
$$

becomes an MMP with scaling $C^{\prime}$ that is the pullback of $C$ to $X^{\prime}$. Hence the termination of flips follows from the case of algebraically closed fields. The argument in [Bir12, Remark 2.9] is working on the characteristic zero, but it also works in our setting since the small $\mathbb{Q}$-factorialization exists by [Bir, Theorem 1.6] and the MMP necessary in the argument is known to exist by [BW, Theorem 1.6].

We can drop the projectivity assumption on $Z$.

Theorem 2.13 (Relative MMP). Let $k$ be a perfect field of characteristic $p>5$, and let $(X, \Delta)$ be a $\mathbb{Q}$-factorial klt threefold over $k$, equipped with a projective morphism $f: X \rightarrow Z$ to a quasi-projective variety $Z$ over $k$. Then, we can run some $\left(K_{X}+\Delta\right)-M M P$ over $Z$ and it terminates. More precisely, there exists a sequence

$$
(X, \Delta)=:\left(X_{1}, \Delta_{1}\right) \rightarrow\left(X_{2}, \Delta_{2}\right) \rightarrow \cdots \rightarrow\left(X_{N}, \Delta_{N}\right)
$$

consisting of divisorial contractions over $Z$ and flips over $Z$ such that $K_{X_{N}}+$ $\Delta_{N}$ is nef over $Z$ or $\left(X_{N}, \Delta_{N}\right)$ has a Mori fiber space structure over $Z$.

Proof. We may assume $f_{*} \mathcal{O}_{X}=\mathcal{O}_{Z}$. We can construct a projective $\mathbb{Q}$ factorial klt compactification $\bar{f}:(\bar{X}, \bar{\Delta}) \rightarrow \bar{Z}$ of $f:(X, \Delta) \rightarrow Z$ in the following way. Let $X^{\prime} \rightarrow Z^{\prime}$ be an arbitrary projective compactification of $X \rightarrow Z$, where $X^{\prime}$ and $Z^{\prime}$ are projective normal varieties. Set $\Delta^{\prime}$ to be the closure of $\Delta$ (note here that $K_{X^{\prime}}+\Delta^{\prime}$ may not be $\mathbb{Q}$-Cartier). Let $\varphi^{\prime}: Y^{\prime} \rightarrow$ $X^{\prime}$ be a resolution of singularities such that the support of $\varphi^{\prime-1}\left(\operatorname{Supp}\left(\Delta^{\prime}\right)\right) \cup$ $\operatorname{Ex}\left(\varphi^{\prime}\right)$ is a simple normal crossing divisor. Set $Y:=\varphi^{\prime-1}(X)$ and write

$$
\varphi:=\left.\varphi^{\prime}\right|_{Y}: Y \rightarrow X \text {. }
$$

We can write

$$
K_{Y}+\varphi_{*}^{-1} \Delta+(1-\epsilon) E=\varphi^{*}\left(K_{X}+\Delta\right)+E^{\prime},
$$


where $E$ is the sum of the $\varphi$-exceptional prime divisors, $\epsilon$ is a rational number with $0<\epsilon<1$, and $E^{\prime}$ is a $\varphi$-exceptional effective $\mathbb{Q}$-divisor satisfying $\operatorname{Supp} E^{\prime}=\operatorname{Ex}(\varphi)$. For the closure $\Gamma$ of $\varphi_{*}^{-1} \Delta+(1-\epsilon) E$ in $Y^{\prime}$, it follows from the choice of $\varphi^{\prime}$ that $\left(Y^{\prime}, \Gamma\right)$ is klt. We run a $\left(K_{Y^{\prime}}+\Gamma\right)$-MMP over $X^{\prime}$ (Theorem 2.12), and set $(\bar{X}, \bar{\Delta})$ to be the end result. Then $(\bar{X}, \bar{\Delta})$ is a $\mathbb{Q}$-factorial klt pair. Further, by the negativity lemma and the $\mathbb{Q}$-factoriality of $X$, the pair $(\bar{X}, \bar{\Delta})$ is a compactification of $(X, \Delta)$.

We run a $\left(K_{\bar{X}}+\bar{\Delta}\right)$-MMP over $\bar{Z}$ with scaling of some ample divisor and it terminates by Theorem 2.12. Then the assertion follows by restricting this MMP to $Z$.

Theorem 2.14 (Q্Q-factorialization). Let $k$ be a perfect field of characteristic $p>5$, and let $(X, \Delta)$ be a klt threefold over $k$. Then there exists a small projective birational morphism $f: Y \rightarrow X$ such that $Y$ is $\mathbb{Q}$-factorial.

Proof. Let $g: Z \rightarrow X$ be a $\log$ resolution and let $E$ be the sum of the $g$-exceptional prime divisors. For a small $\epsilon>0$, we can run a $\left(K_{Z}+g_{*}^{-1} \Delta+\right.$ $(1-\epsilon) E$ )-MMP over $X$, and it terminates with a $\mathbb{Q}$-factorial model $Y$ (Theorem 2.13).

Since $E$ is contracted by this MMP by the negativity lemma, $Y \rightarrow X$ turns out to be small.

2.4. Special fibers of log Fano contractions. The following proposition shall be used repeatedly in this paper. This proposition allows us to assume that a closed fiber of a log Fano contraction is a log Fano variety in many situations.

Proposition 2.15. Let $k$ be a perfect field of characteristic $p>5$. Let $f: X \rightarrow Z$ be a projective morphism of normal quasi-projective varieties over $k$ with the following properties.

- $(X, \Delta)$ is a klt threefold.

- $0<\operatorname{dim} Z \leq 3$.

- $f_{*} \mathcal{O}_{X}=\mathcal{O}_{Z}$ and $-\left(K_{X}+\Delta\right)$ is $f$-nef and $f$-big.

Fix a closed point $z \in Z$. Then there exists a commutative diagram of quasi-projective normal varieties

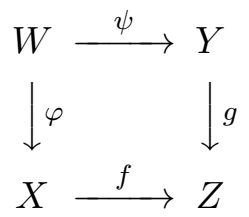

and an effective $\mathbb{Q}$-divisor $\Delta_{Y}$ on $Y$ which satisfy the following properties.

(1) $\left(Y, \Delta_{Y}\right)$ is a $\mathbb{Q}$-factorial plt threefold and $-\left(K_{Y}+\Delta_{Y}\right)$ is g-ample.

(2) $-\left\llcorner\Delta_{Y}\right\lrcorner$ is g-nef and $g^{-1}(z)_{\mathrm{red}}=\left\llcorner\Delta_{Y}\right\lrcorner$.

(3) $W$ is a smooth threefold, and both of $\varphi$ and $\psi$ are projective birational morphisms.

(4) If $X=Z$, then $g(\operatorname{Ex}(g))$ is a finite set. More precisely, $g$ is isomorphic outside $z$ and the non- $\mathbb{Q}$-factorial points on $Z$.

In particular, $\left\llcorner\Delta_{Y}\right\lrcorner$ is a surface of Fano type by Theorem 2.11 and adjunction (See Definition 4.2 for the definition of Fano type). 
Proof. We may assume that $-\left(K_{X}+\Delta\right)$ is $f$-ample. Let $h: V \rightarrow X$ be a $\log$ resolution of $(X, \Delta)$. Possibly replacing $V$ with a higher model, we can find a Cartier divisor $D_{Z}$ on $Z$ such that

$$
0 \sim_{Z} h^{*} f^{*} D_{Z}=M+F
$$

where $M$ is a base point free Cartier divisor and $F=\sum f_{i} E_{i}$ is an effective divisor with $\operatorname{Supp} F=\operatorname{Supp} V_{z}$, where $V_{z}$ is the fiber of $V \rightarrow Z$ over $z$ (note that $F$ may not be $h$-exceptional). We may assume that $\operatorname{Supp}\left(h^{-1}(\Delta) \cup F \cup\right.$ $\operatorname{Ex}(h))$ is simple normal crossing. We can write

$$
K_{V}+h_{*}^{-1} \Delta=h^{*}\left(K_{X}+\Delta\right)+\sum a_{i} E_{i}
$$

with $a_{i}>-1$. Since $-h^{*}\left(K_{X}+\Delta\right)$ is nef and big over $Z$, we can write $-h^{*}\left(K_{X}+\Delta\right)=A+G$, where $A$ is an ample $\mathbb{Q}$-divisor over $Z$ and $G$ is an effective $h$-exceptional $\mathbb{Q}$-divisor. Let $G=\sum_{i} g_{i} E_{i}$ be the decomposition by prime divisors of $G$. Then we can take a small positive rational number $\epsilon$ such that $a_{i}-\epsilon g_{i}>-1$ for any $i$. Then, we have

$$
K_{V}+h_{*}^{-1} \Delta+\epsilon A-(1-\epsilon) h^{*}\left(K_{X}+\Delta\right)=\sum\left(a_{i}-\epsilon g_{i}\right) E_{i} .
$$

Since $M+F \sim_{Z} 0$, we can find $\lambda \in \mathbb{Q}_{>0}$ with

$K_{V}+h_{*}^{-1} \Delta+\left(\epsilon A-(1-\epsilon) h^{*}\left(K_{X}+\Delta\right)+\lambda M\right) \sim_{\mathbb{Q}, Z} \sum\left(a_{i}-\epsilon g_{i}-\lambda f_{i}\right) E_{i}$, such that $a_{i}-\epsilon g_{i}-\lambda f_{i} \geq-1$ holds for any $i$ and at least one index attains -1 .

Let $S_{V}$ be a prime divisor which attains -1 in the above. Since $\operatorname{Supp} F=$ Supp $V_{z}$, it follows that $S_{V} \subset V_{z}$. Let $\left\{E_{i}\right\}_{i \in I^{\prime}}$ be the set of the other prime divisors contained in $V_{z} \cup \operatorname{Ex}(h)$. Since $\epsilon A-(1-\epsilon) h^{*}\left(K_{X}+\Delta\right)+\lambda M$ is ample over $Z$, we may find an ample $\mathbb{Q}$-divisor $A^{\prime}$ such that

$$
K_{V}+h_{*}^{-1} \Delta+A^{\prime}+S_{V}+\sum_{i \in I^{\prime}} E_{i} \sim_{\mathbb{Q}, Z} \sum_{i \in I^{\prime}} b_{i} E_{i}
$$

with $b_{i}>0$. Note that $S_{V}$ and $\sum_{i \in I^{\prime}} b_{i} E_{i}$ may not be $h$-exceptional, but that $\operatorname{Supp} \sum_{i \in I^{\prime}} b_{i} E_{i}$ contains all the $h$-exceptional prime divisors whose images in $X$ are not contained in $X_{z}$. We can assume that $A^{\prime}$ is effective and $\left\llcorner A^{\prime}\right\lrcorner=0$, and that $h_{*}^{-1} \Delta+A^{\prime}+S_{V}+\sum_{i \in I^{\prime}} E_{i}$ has a simple normal crossing support. Set $B_{V}:=h_{*}^{-1} \Delta+A^{\prime}$, which is big.

We shall run an MMP three times to get a desired $g: Y \rightarrow Z$.

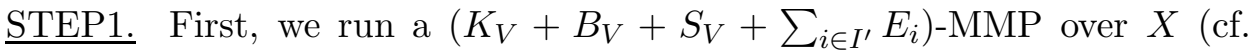
Lemma 2.2, Theorem 2.9, and Theorem 2.10). Since this MMP is also $\left(\sum_{i \in I^{\prime}} b_{i} E_{i}\right)$-MMP, the curves contracted by this MMP is contained in

$$
\bigcup_{i \in I^{\prime}} \operatorname{Supp} E_{i} \text {. }
$$

Hence this MMP terminates by the special termination (Theorem 2.6), and ends with a minimal model $\left(V^{\prime}, B_{V^{\prime}}+S_{V^{\prime}}+\sum_{i \in I^{\prime}} E_{i}^{\prime}\right)$ over $X$, that is

$$
K_{V^{\prime}}+B_{V^{\prime}}+S_{V^{\prime}}+\sum_{i \in I^{\prime}} E_{i}^{\prime} \sim_{\mathbb{Q}, Z} \sum_{i \in I^{\prime}} b_{i} E_{i}^{\prime} .
$$

is nef over $X$. Note that $E_{i}^{\prime}=0$ if $E_{i}$ is contracted by this MMP. Since this MMP contracts a curve contained in $\bigcup_{i \in I^{\prime}}$ Supp $E_{i}$, this MMP does not 
contract $S_{V}$. We can also see that $\operatorname{Supp}\left(\sum b_{i} E_{i}^{\prime}\right) \subset V_{z}^{\prime}$ by the negativity lemma for $V^{\prime} \backslash V_{z}^{\prime}$ over $X \backslash X_{z}$.

$\underline{\text { STEP2. }}$ Second, we run a $\left(K_{V^{\prime}}+B_{V^{\prime}}+S_{V^{\prime}}+\sum_{i \in I^{\prime}} E_{i}^{\prime}\right)$-MMP over $Z$. By the same reason as in STEP1, this MMP terminates and ends with a minimal model $\left(V^{\prime \prime}, B_{V^{\prime \prime}}+S_{V^{\prime \prime}}+\sum_{i \in I^{\prime}} E_{i}^{\prime \prime}\right)$ over $Z$, that is,

$$
K_{V^{\prime \prime}}+B_{V^{\prime \prime}}+S_{V^{\prime \prime}}+\sum_{i \in I^{\prime}} E_{i}^{\prime \prime} \sim_{\mathbb{Q}, Z} \sum_{i \in I^{\prime}} b_{i} E_{i}^{\prime \prime}
$$

is nef over $Z$. Further, this MMP does not contract $S_{V^{\prime}}$.

We shall show that $\sum_{i \in I^{\prime}} b_{i} E_{i}^{\prime \prime} \sim_{\mathbb{Q}, Z} 0$. Let $G_{Z}$ be an effective Cartier divisor on $Z$ passing through $z$, and let $G_{V^{\prime \prime}}$ be its pull-back to $V^{\prime \prime}$. Since $\operatorname{Supp}\left(\sum b_{i} E_{i}^{\prime}\right) \subset V_{z}^{\prime}$, there exists $\mu \geq 0$ such that

$$
-\mu G_{V^{\prime \prime}}+\sum_{i \in I^{\prime}} b_{i} E_{i}^{\prime \prime} \leq 0
$$

and at least one coefficient of $E_{i}^{\prime \prime}$ in $-\mu G_{V^{\prime \prime}}+\sum_{i \in I^{\prime}} b_{i} E_{i}^{\prime \prime}$ is zero. If

$$
-\mu G_{V^{\prime \prime}}+\sum_{i \in I^{\prime}} b_{i} E_{i}^{\prime \prime} \sim_{\mathbb{Q}, Z} \sum_{i \in I^{\prime}} b_{i} E_{i}^{\prime \prime} \varkappa_{\mathbb{Q}, Z} 0,
$$

there exists a prime divisor $E_{i_{0}}^{\prime \prime}$ whose coefficient in $-\mu G_{V^{\prime \prime}}+\sum_{i \in I^{\prime}} b_{i} E_{i}^{\prime \prime}$ is zero but one of the prime divisors that meet $E_{i_{0}}^{\prime \prime}$ but are different from $E_{i_{0}}^{\prime \prime}$ has negative coefficient. Then a general curve on $E_{i_{0}}^{\prime \prime}$ has negative intersection number with $-\mu G_{V^{\prime \prime}}+\sum_{i \in I^{\prime}} b_{i} E_{i}^{\prime \prime}$, and it contradicts the nefness of $-\mu G_{V^{\prime \prime}}+$ $\sum_{i \in I^{\prime}} b_{i} E_{i}^{\prime \prime}$ over $Z$. Hence it follows that $\sum_{i \in I^{\prime}} b_{i} E_{i}^{\prime \prime} \sim_{\mathbb{Q}, Z} 0$ by contradiction.

STEP3. Third, we run a $\left(K_{V^{\prime \prime}}+B_{V^{\prime \prime}}+\frac{1}{2} S_{V^{\prime \prime}}+\sum_{i \in I^{\prime}} E_{i}^{\prime \prime}\right)$-MMP over $Z$. Since $\sum b_{i} E_{i}^{\prime \prime} \sim_{\mathbb{Q}, Z} 0$, this MMP can be considered as an MMP for a klt pair, hence we may run this MMP and it terminates. By

$$
K_{V^{\prime \prime}}+B_{V^{\prime \prime}}+\frac{1}{2} S_{V^{\prime \prime}}+\sum_{i \in I^{\prime}} E_{i}^{\prime \prime} \sim_{\mathbb{Q}, Z}-\frac{1}{2} S_{V^{\prime \prime}},
$$

this MMP is $S_{V^{\prime \prime}}$-positive, hence does not contract $S_{V^{\prime \prime}}$. Since $S_{V^{\prime \prime}}$ is contained in the fiber $V_{z}^{\prime \prime}$ over $z$, we can write $-\frac{1}{2} S_{V^{\prime \prime}} \sim_{\mathbb{Q}, Z} L$ for some effective $\mathbb{Q}$-divisor $L$ on $V^{\prime \prime}$. Therefore, the end result $\left(Y, B_{Y}+\frac{1}{2} S+\sum_{i \in I^{\prime}} E_{i, Y}\right)$ is a minimal model equipped with $g: Y \rightarrow Z$. Since $-S$ is $g$-nef and $S \subset g^{-1}(z)_{\text {red }}$, it follows that $S=g^{-1}(z)_{\text {red. }}$. In particular, all the prime divisors in $\sum_{i \in I^{\prime}} E_{i}^{\prime \prime}$ are contracted in this MMP. Therefore, we obtain

$$
K_{Y}+B_{Y}+S \sim_{\mathbb{Q}, Z} 0 .
$$

Since $B_{V}$ is big, so is $B_{Y}$. We can find a $\mathbb{Q}$-divisor $B \geq 0$ such that $(Y, S+B)$ is plt and $-\left(K_{Y}+S+B\right)$ is ample. It completes the proof of (1)-(3).

Finally, we prove (4). Suppose $X=Z$. Then STEP2 is unnecessary. In STEP1, all the exceptional divisors of $V^{\prime} \rightarrow X$ are contained in the fiber $V_{z}^{\prime}$ (recall that we saw $\left.\operatorname{Supp}\left(\sum b_{i} E_{i}^{\prime}\right) \subset V_{z}^{\prime}\right)$. Then $V^{\prime} \rightarrow X$ turns out to be isomorphic outside $\left\{z, x_{1}, \cdots, x_{r}\right\}$, where $x_{1}, \cdots, x_{r}$ are the non- $\mathbb{Q}$-factorial points of $X=Z$. Therefore, the result $Y \rightarrow X=Z$ is also isomorphic outside $\left\{z, x_{1}, \cdots, x_{r}\right\}$. 
Remark 2.16. For the case $X=Z$ in Proposition 2.15, $Y$ is very similarly constructed as the Kollár component in [Xu14, Lemma 1].

Proposition 2.15 holds except for (4) in any dimension if we assume the existence of log resolution and an MMP with scaling.

2.5. Witt vectors. We briefly recall the definition of the ring of Witt vectors. For more details we refer the reader [Ser79, II, Section 6].

Let $k$ be a perfect field of characteristic $p>0$. We define a functor $W$ from the category of $\mathbb{F}_{p}$-algebras to the category of $\mathbb{Z}$-algebras:

$$
W: \mathbf{A} \mathbf{l g}_{/ \mathbb{F}_{p}} \rightarrow \mathbf{A l g} \lg _{/ \mathbb{Z}} ; \quad A \mapsto W A
$$

Although we can define $W A$ for any ring $A$, we restrict ourselves to treat $\mathbb{F}_{p^{-}}$ algebras. Firstly, as a set, we define $W A$ to be the product $A^{\mathbb{N}}$ of countably many copies of $A$ :

$$
W A=A \times A \times A \times \cdots=\left\{\left(a_{0}, a_{1}, a_{2}, \ldots\right) \mid a_{i} \in A\right\} .
$$

The zero and the unity element are

$$
0_{W A}=\left(0_{A}, 0_{A}, 0_{A}, \ldots\right), \quad 1_{W A}=\left(1_{A}, 0_{A}, 0_{A}, \ldots\right) .
$$

We define the ring structure as follows: For two vectors $a=\left(a_{0}, a_{1}, \ldots\right), b=$ $\left(b_{0}, b_{1}, \ldots\right)$, we set

$$
\begin{aligned}
a+b & :=\left(S_{0}\left(a_{0}, b_{0}\right), S_{1}\left(a_{0}, a_{1}, b_{0}, b_{1}\right), S_{2}\left(a_{0}, a_{1}, a_{2}, b_{0}, b_{1}, b_{2}\right) \ldots\right), \\
a \cdot b & :=\left(P_{0}\left(a_{0}, b_{0}\right), P_{1}\left(a_{0}, a_{1}, b_{0}, b_{1}\right), P_{2}\left(a_{0}, a_{1}, a_{2}, b_{0}, b_{1}, b_{2}\right) \ldots\right),
\end{aligned}
$$

where $S_{i}, P_{i} \in \mathbb{Z}\left[X_{0}, \ldots, X_{i}, Y_{0}, \ldots, Y_{i}\right]$ are the unique polynomials satisfying the following law:

- When we set polynomials $w_{n}$ as $w_{n}\left(X_{0}, \cdots, X_{n}\right):=\sum_{0 \leq i \leq n} p^{i} X_{i}^{p-i}$ for each $n \geq 0, S_{i}$ and $P_{i}$ satisfy the following equations

$$
\begin{aligned}
& w_{n}\left(S_{0}, \ldots, S_{n}\right)=w_{n}\left(X_{0}, \ldots, X_{n}\right)+w_{n}\left(Y_{0}, \ldots, Y_{n}\right), \\
& w_{n}\left(P_{0}, \ldots, P_{n}\right)=w_{n}\left(X_{0}, \ldots, X_{n}\right) w_{n}\left(Y_{0}, \ldots, Y_{n}\right)
\end{aligned}
$$

for each $n \geq 0$.

We have, for example, $W\left(\mathbb{F}_{p}\right)=\mathbb{Z}_{p}$. In general, $W(k)$ is a complete discrete valuation ring of mixed characteristic with $W(k) / p W(k)=k$. In particular, $W(k)_{\mathbb{Q}}:=W(k) \otimes_{\mathbb{Z}} \mathbb{Q}$ is a field.

For a ring homomorphism $\varphi: A \rightarrow B$, we define a ring homomorphism $W \varphi$ by

$$
W \varphi: W A \rightarrow W B ; \quad\left(a_{0}, a_{1}, a_{2}, \ldots\right) \mapsto\left(\varphi\left(a_{0}\right), \varphi\left(a_{1}\right), \varphi\left(a_{2}\right), \ldots\right) .
$$

We note that if $\varphi$ is injective (resp. surjective), so is $W \varphi$. In particular, if $A$ is a nonzero $k$-algebra, then the natural ring homomorphism $k \rightarrow A$ is injective and so we have an injective ring homomorphism $W(k) \rightarrow W A$. Hence $W A$ has a $W(k)$-algebra structure.

For $n \geq 1$, we can also define a ring $W_{n} A$ which is equal to $A^{n}$ as a set and with the same operation as $W A$. We have a natural projection

$$
R: W_{n+1} A \rightarrow W_{n} A ; \quad\left(a_{0}, \ldots, a_{n-1}, a_{n}\right) \mapsto\left(a_{0}, \ldots, a_{n-1}\right) .
$$

Then the ring $W A$ is isomorphic to the projective limit $W A=\lim _{\longleftarrow} W_{n} A$. In general, $W_{n}(k)$ is an Artinian ring. 
We can define maps $V$ and $F$ as follows:

$$
\begin{aligned}
& V: W_{n} A \rightarrow W_{n+1} A ; \quad\left(a_{0}, a_{1}, \ldots, a_{n-1}\right) \mapsto\left(0, a_{0}, a_{1}, \ldots, a_{n-1}\right), \\
& F: W_{n} A \rightarrow W_{n} A ; \quad\left(a_{0}, a_{1}, \ldots, a_{n-1}\right) \mapsto\left(a_{0}^{p}, a_{1}^{p}, \ldots, a_{n-1}^{p}\right) .
\end{aligned}
$$

It is well-known that $F$ is a homomorphism of rings and that $V$ is a homomorphism of additive groups. We can also define $V$ and $F$ as endomorphisms on $W A$. We also note that $p a:=\underbrace{a+\cdots+a}_{p \text { times }}=V F(a)=F V(a)$ holds for $a \in W A$.

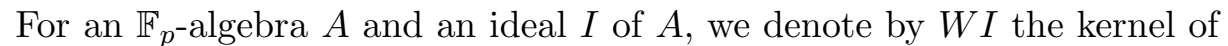
the induced surjective ring homomorphism $W A \rightarrow W(A / I)$. As a set, we may write

$$
W I=\left\{\left(a_{0}, a_{1}, a_{2}, \ldots\right) \in W A \mid a_{i} \in I\right\} .
$$

We may also define $W_{n} I$ as above.

The ring of Witt vectors can be generalized to the sheaf of Witt vectors (cf. [BBE07, Section 2], [Ill79, Section 1]). For a $k$-scheme $X$, we define the presheaf $W \mathcal{O}_{X}$ on $X$ as

$$
\left(W \mathcal{O}_{X}\right)(U):=W\left(\mathcal{O}_{X}(U)\right)
$$

for any open subset $U \subset X$. This presheaf is already a sheaf. For a quasicoherent ideal sheaf $I \subset \mathcal{O}_{X}$, we can also define a sheaf $W I$ on $X$ similarly. Further, we can also define $W_{n} \mathcal{O}_{X}$ and $W_{n} I$ and $R, V, F$ similarly. We emphasize here that $W_{n} \mathcal{O}_{X}, W_{n} I, W \mathcal{O}_{X}$ and $W I$ do not have an $\mathcal{O}_{X^{-}}$ module structure if $n \neq 1$, but they are sheaves of abelian groups.

For a $k$-scheme $X$, we can define a ringed space $W_{n} X$ as follows

$$
W_{n} X:=\left(|X|, W_{n} \mathcal{O}_{X}\right) .
$$

Since $W_{n} X=\operatorname{Spec} W_{n} \mathcal{O}_{X}$, it has a $W_{n}(k)$-scheme structure. If $f: X \rightarrow$ $Y$ is a separated (resp. finite type, proper) morphism of $k$-schemes, then $W_{n} f: W_{n} X \rightarrow W_{n} Y$ is a separated (resp. finite type, proper) morphism of $W_{n}(k)$-schemes. We note here that for an ideal sheaf $I \subset \mathcal{O}_{X}$, the higher direct image sheaf $R^{i}\left(W_{n} f\right)_{*}\left(W_{n} I\right)$ coincides with $R^{i} f_{*}\left(W_{n} I\right)$, and we often use the latter notation.

Lastly, we introduce the notation $W I_{\mathbb{Q}}$ and $R^{i} f_{*}\left(W I_{\mathbb{Q}}\right)$ following [CR12, Subsection 3.7]. For a $k$-scheme $X$, we denote by $\mathcal{A}(X)$ the abelian category of the sheaves of abelian groups on $X$. We define

$$
\mathcal{A}_{\mathrm{b} \text {-tor }}(X):=\left\{F \in \operatorname{ob}(\mathcal{A}(X)) \mid \exists n \in \mathbb{Z}_{>0}: n \cdot \operatorname{id}_{F}=0\right\}
$$

as a full subcategory of $\mathcal{A}(X)$. Then the quotient category $\mathcal{A}(X)_{\mathbb{Q}}:=$ $\mathcal{A}(X) / \mathcal{A}_{\mathrm{b} \text {-tor }}(X)$ exists and is an abelian category. We denote the projection functor by

$$
q: \mathcal{A}(X) \rightarrow \mathcal{A}(X)_{\mathbb{Q}} .
$$

We also use the notation $(-)_{\mathbb{Q}}:=q(-)$ for simplicity. For example, for a coherent ideal sheaf $I$ on $X, W I_{\mathbb{Q}}$ is defined by $W I_{\mathbb{Q}}=q(W I)$ and it is an object of $\mathcal{A}(X)_{\mathbb{Q}}$ (hence $W I_{\mathbb{Q}}$ is not a sheaf). For a morphism $f: X \rightarrow Y$ of $k$-schemes, the functor $f_{*}: \mathcal{A}(X) \rightarrow \mathcal{A}(Y)$ induces the functor on the quotient categories:

$$
f_{*}: \mathcal{A}(X)_{\mathbb{Q}} \rightarrow \mathcal{A}(Y)_{\mathbb{Q}},
$$


and the right derived functor

$$
R^{i} f_{*}: \mathcal{A}(X)_{\mathbb{Q}} \rightarrow \mathcal{A}(Y)_{\mathbb{Q}}
$$

for $i \geq 0$. When $Y=\operatorname{Spec} k$, we write $H^{i}\left(X, F_{\mathbb{Q}}\right)$ instead of $R^{i} f_{*} F_{\mathbb{Q}}$ for a sheaf $F$ on $X$.

We give here some remarks on the notation $R^{i} f_{*} W I_{\mathbb{Q}}$.

Remark 2.17. (1) In [CR12], $R^{i} f_{*} W I_{\mathbb{Q}}$ is considered as a object in the category $\widehat{\mathrm{dRW}}_{X, \mathbb{Q}}:=\widehat{\mathrm{dRW}}_{X} / \widehat{\mathrm{dRW}}_{X, \text { b-tor. However, for our aim, it }}$ is sufficient to work on $\mathcal{A}(X)_{\mathbb{Q}}$ described as above.

(2) Let $f: X \rightarrow Y$ be a morphism of separated schemes of finite type over $k$ and let $I$ be a coherent ideal sheaf on $X$. Then by [CR12, Proposition 3.7.7], we have that

$$
R^{i} f_{*}\left(W I_{\mathbb{Q}}\right) \simeq\left(R^{i} f_{*}(W I)\right)_{\mathbb{Q}}
$$

for each non-negative integer $i$, in the category $\mathcal{A}(Y)_{\mathbb{Q}}$.

(3) Note that $R^{i} f_{*}\left(W I_{\mathbb{Q}}\right)$ differs from the following sheaf:

$$
R^{i} f_{*}(W I) \otimes_{\mathbb{Z}} \mathbb{Q}: U \mapsto \Gamma\left(U, R^{i} f_{*}(W I)\right) \otimes_{\mathbb{Z}} \mathbb{Q} .
$$

First of all, $R^{i} f_{*}\left(W I_{\mathbb{Q}}\right)$ is not a sheaf on $Y$ (it is an object in $\left.\mathcal{A}(Y)_{\mathbb{Q}}\right)$. However, by $(2)$ in this remark, the condition $R^{i} f_{*}\left(W I_{\mathbb{Q}}\right)=0$ is equivalent to the condition that there exists a positive integer $N$ such that $N \cdot R^{i} f_{*}(W I)=0$. On the other hand, $R^{i} f_{*}(W I) \otimes_{\mathbb{Z}} \mathbb{Q}=0$ if and only if for any open subset $U$ of $Y$ and section $s \in \Gamma\left(U, R^{i} f_{*}(W I)\right)$, there exists a positive integer $N_{s}$ such that $N_{s} \cdot s=0$. In particular, $R^{i} f_{*}\left(W I_{\mathbb{Q}}\right)=0$ implies $R^{i} f_{*}(W I) \otimes_{\mathbb{Z}} \mathbb{Q}=0$.

(4) The notation $R^{i} f_{*}\left(W I_{K}\right)$ used in [BBE07] is the same as $R^{i} f_{*}(W I) \otimes_{\mathbb{Z}}$ $\mathbb{Q}$. However, some of their results (e.g. [BBE07, 2.1, 2.2, 2.4]) are still valid for $R^{i} f_{*}\left(W I_{\mathbb{Q}}\right)$. See the proof of Proposition 2.23.

(5) If $Z$ is a proper scheme over $k$, then $H^{i}\left(Z, W \mathcal{O}_{Z, \mathbb{Q}}\right)=0$ if and only if $H^{i}\left(Z, W \mathcal{O}_{Z}\right) \otimes_{\mathbb{Z}} \mathbb{Q}=0$. Indeed, this equivalence follows from (3) in this remark and the fact that $H^{i}\left(Z, W \mathcal{O}_{Z}\right)$ is a finitely generated $W_{\sigma}[[V]]$-module (cf. the proof of Proposition 2.10 in [BBE07]).

2.6. Fundamental properties of Witt vector cohomologies. In this subsection, we summarize some basic properties of Witt vector cohomologies.

Lemma 2.18. Let $f: X \rightarrow Y$ be a morphism between separated schemes of finite type over a perfect field $k$ of characteristic $p>0$. Let $I$ be a coherent ideal sheaf on $X$. Then, the following assertions hold.

(1) $R^{i} \lim _{n}\left(f_{*} W_{n} I\right)=0$ for every $i \geq 1$.

(2) $R^{i} \varliminf_{n}\left(R^{j} f_{*} W_{n} I\right)=0$ for every $i \geq 2$ and every $j \geq 0$.

Proof. It is sufficient to show that the following two conditions hold for any affine open subset $U \subset X$ (cf. [CR12, Lemma 1.5.1]):

- $H^{i}\left(U, R^{j} f_{*}\left(W_{n} I\right)\right)=0$ for any $i, n \geq 1$ and $j \geq 0$.

- $H^{0}\left(U, f_{*}\left(W_{n+1} I\right)\right) \rightarrow H^{0}\left(U, f_{*}\left(W_{n} I\right)\right)$ is surjective for any $n \geq 1$.

We note that $W_{n} I$ becomes a coherent ideal sheaf on a scheme $W_{n} X$ of finite type over an Artinian ring $W_{n}(k)$. Therefore, $R^{j} f_{*}\left(W_{n} I\right)=R^{j}\left(W_{n} f\right)_{*}\left(W_{n} I\right)$ 
is also a quasi-coherent sheaf on $W_{n} Y$. Since $R^{j} f_{*}\left(W_{n} I\right)$ is a quasi-coherent sheaf on the Noetherian scheme $W_{n} Y$, we see

$$
H^{i}\left(U, R^{j} f_{*}\left(W_{n} I\right)\right)=0
$$

for any affine open subset $U \subset X$ and $i>0$.

The map $H^{0}\left(U, f_{*}\left(W_{n+1} I\right)\right) \rightarrow H^{0}\left(U, f_{*}\left(W_{n} I\right)\right)$ is the same as

$$
W_{n+1}\left(I\left(f^{-1}(U)\right)\right) \rightarrow W_{n}\left(I\left(f^{-1}(U)\right)\right),
$$

and it is clearly surjective.

The following two lemmas are consequences of the above lemma.

Lemma 2.19. Let $f: X \rightarrow Y$ be a morphism between separated schemes of finite type over a perfect field $k$ of characteristic $p>0$. Let $I$ be a coherent ideal sheaf on $X$ satisfying $R^{i} f_{*} I=0$ for every $i>0$. Then $R^{i} f_{*}(W I)=0$ holds for every $i>0$.

Proof. First we note that $R^{i} f_{*}\left(W_{n} I\right)=0$ holds for each $i \geq 1$ and $n \geq 1$. This follows from the induction on $n$ using the assumption and the short exact sequence of sheaves of abelian groups:

$$
0 \rightarrow I \stackrel{V^{n}}{\rightarrow} W_{n+1} I \stackrel{R}{\rightarrow} W_{n} I \rightarrow 0 .
$$

Then the assertion follows from the following isomorphisms of complexes in the derived category

$$
\begin{aligned}
& R f_{*}(W I) \cong R f_{*}\left(R{\underset{\leftarrow}{n}}_{\lim _{n}}\left(W_{n} I\right)\right) \cong R{\underset{\leftarrow}{n}}_{\lim }\left(R f_{*}\left(W_{n} I\right)\right) \\
& \cong R{\underset{n}{n}}_{\lim _{n}}\left(f_{*} W_{n} I\right) \cong \varliminf_{n} \lim _{*}\left(f_{*} W_{n} I\right) \text {. }
\end{aligned}
$$

The first and fourth isomorphisms follow from Lemma 2.18 (1), and the third follows from the above remark. For the second isomorphism, note that $f_{*} \circ \lim _{n}=\lim _{n} \circ f_{*}$ as a functor.

Lemma 2.20. Let $f: X \rightarrow Y$ be a morphism between separated schemes of finite type over a perfect field $k$ of characteristic $p>0$. Let $I$ be a coherent ideal sheaf on $X$. Then, $R^{i} f_{*}(W I)=0$ holds for every $i>m:=$ $\max _{y \in Y} \operatorname{dim} f^{-1}(y)$.

Proof. First we note that $R^{i} f_{*}\left(W_{n} I\right)=0$ holds for $i>m$ since the sheaf $W_{n} I$ is a quasi-coherent sheaf on the Noetherian scheme $W_{n} X$.

Since $R^{i} \lim _{n}\left(W_{n} I\right)=0$ for $i>0$ (Lemma $2.18(1)$ ), we obtain

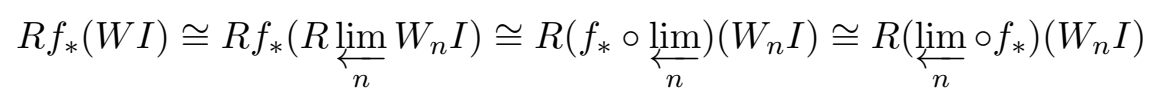

as complexes in the derived category, and hence we have $R^{i} f_{*}(W I) \cong$ $R^{i}\left(\lim _{n} \circ f_{*}\right)\left(W_{n} I\right)$ for each $i$. Thus, we obtain the following spectral sequence:

$$
\begin{aligned}
E_{2}^{i, j} & :=R^{i} \lim _{n} R^{j} f_{*}\left(W_{n} I\right) \\
& \Longrightarrow E^{i+j}:=R^{i+j}\left({\underset{\swarrow}{n}}_{\lim _{n}} \circ f_{*}\right)\left(W_{n} I\right) \cong R^{i+j} f_{*}(W I) .
\end{aligned}
$$


By Lemma 2.18 (2), we see $E_{2}^{i, j}=0$ for $i \geq 2$. Then, for each $j$, we obtain the following exact sequence

$$
0 \rightarrow R^{1}{\underset{\leftarrow}{n}}_{\lim } R^{j-1} f_{*}\left(W_{n} I\right) \rightarrow R^{j} f_{*}(W I) \rightarrow{\underset{\varliminf}{n}}_{\lim } R^{j} f_{*}\left(W_{n} I\right) \rightarrow 0 .
$$

Therefore $R^{j} f_{*}(W I)=0$ holds if $j \geq m+2$.

We show $R^{m+1} f_{*}(W I)=0$. By the above short exact sequence, we obtain

$$
R^{1}{\underset{\leftarrow}{n}}_{\lim ^{m}} R_{*}\left(W_{n} I\right) \cong R^{m+1} f_{*}(W I) .
$$

In order to show $R^{1} \lim _{\longleftarrow} R^{m} f_{*}\left(W_{n} I\right)=0$, it is sufficient to check the surjectivity of the map

$$
R^{m} f_{*}\left(W_{n+1} I\right) \rightarrow R^{m} f_{*}\left(W_{n} I\right) .
$$

It can be confirmed by the short exact sequence

$$
0 \rightarrow I \stackrel{V^{n}}{\rightarrow} W_{n+1} I \stackrel{R}{\rightarrow} W_{n} I \rightarrow 0,
$$

and the vanishing $R^{m+1} f_{*} I=0$.

The vanishing of $R^{i} f_{*}(W I)$ implies the vanishing of $R^{i} f_{*}\left(W I_{\mathbb{Q}}\right)$.

Lemma 2.21. Let $f: X \rightarrow Y$ be a morphism of separated schemes of finite type over a perfect field $k$ of characteristic $p>0$. Let $I$ be a coherent ideal sheaf on $X$ and let $i$ be a non-negative integer. If $R^{i} f_{*}(W I)=0$ holds, then $R^{i} f_{*}\left(W I_{\mathbb{Q}}\right)=0$ holds.

Proof. This easily follows from $R^{i} f_{*}\left(W I_{\mathbb{Q}}\right) \simeq R^{i} f_{*}(W I)_{\mathbb{Q}}$ by Remark 2.17.

One of the advantages to consider the Witt vector cohomology is from the following lemma which states that the Witt vector cohomology does not change under a finite universal homeomorphism.

Lemma 2.22. Let $f: X \rightarrow Y$ be a proper surjective morphism of separated schemes of finite type over a perfect field $k$ of characteristic $p>0$. If any fiber of $f$ is geometrically connected, then the natural homomorphism $W \mathcal{O}_{Y, \mathbb{Q}} \rightarrow f_{*} W \mathcal{O}_{X, \mathbb{Q}}$ is an isomorphism.

Proof. Taking the Stein factorization of $f$, we may assume that $f$ is a finite universal homeomorphism.

We may assume that $X$ and $Y$ are reduced by BBE07, Proposition 2.1 (i)] (cf. Remark 2.17). Moreover, we may assume that $X$ and $Y$ are affine and may write $X=\operatorname{Spec} B$ and $Y=\operatorname{Spec} A$. Since the induced ring homomorphism $\varphi: A \rightarrow B$ is injective, $W \varphi: W A \rightarrow W B$ is also injective.

Since $f$ is a finite universal homeomorphism, $B^{p^{e}} \subset A$ holds for some $e \in \mathbb{Z}_{>0}$ (cf. [Kol97, Proposition 6.6]), which implies $p^{e}(W B) \subset \varphi(W A)$. Thus $W \varphi_{\mathbb{Q}}: W A_{\mathbb{Q}} \rightarrow W B_{\mathbb{Q}}$ is an isomorphism.

The following proposition, which is a consequence of a theorem BBE07, Theorem 2.4] by Berthelot, Bloch and Esnault, is also a key property of the Witt vector cohomology. 
Proposition 2.23. Let $f: X \rightarrow Y$ be a proper birational morphism of separated schemes of finite type over a perfect field $k$ of characteristic $p>0$, and let $I \subset \mathcal{O}_{X}$ be a coherent ideal sheaf on $Y$. If $f$ is an isomorphism outside $\operatorname{Zeros}(I) \subset X$, then $R^{i} f_{*}\left(W I_{\mathbb{Q}}\right)=0$ holds for $i>0$.

Proof. Although $R^{i} f_{*}\left(W I_{\mathbb{Q}}\right)$ differs from $R^{i} f_{*}\left(W I_{K}\right)$ appearing in [BBE07] (cf. Remark 2.17), we can apply the same argument as in BBE07, Theorem 2.4]. Indeed, it is shown in [BBE07, Theorem 2.4] that there exists a positive integer $c$ such that for any $i \geq 1$, the map $p^{c}: R^{i} f_{*}(W I) \rightarrow R^{i} f_{*}(W I)$ becomes zero. This shows that $R^{i} f_{*}\left(W I_{\mathbb{Q}}\right) \simeq R^{i} f_{*}(W I)_{\mathbb{Q}}=0$ in the category $\mathcal{A}(Y)_{\mathbb{Q}} \cdot$

For later use, we give two easy lemmas.

Lemma 2.24. Let $k$ be a perfect field of characteristic $p>0$. Let $f: X \rightarrow Y$ be a finite morphism of zero-dimensional schemes which are of finite type over $k$. If the induced map

$$
H^{0}\left(Y, W \mathcal{O}_{Y}\right) \otimes_{\mathbb{Z}} \mathbb{Q} \rightarrow H^{0}\left(X, W \mathcal{O}_{X}\right) \otimes_{\mathbb{Z}} \mathbb{Q}
$$

is an isomorphism, then $f$ is a universal homeomorphism.

Proof. Since $W(A) \times W(B) \cong W(A \times B)$, we may assume that $\# Y=1$. Taking the reduced scheme structures, we may assume that $X$ and $Y$ are reduced. We may also assume that $\# X=1$, since otherwise the induced map on the 0 -th Witt vector cohomologies is clearly not surjective. Therefore, we may write

$$
X=\operatorname{Spec} k_{X}, \quad Y=\operatorname{Spec} k_{Y},
$$

where $k \subset k_{Y} \subset k_{X}$ are finite extensions of fields. It suffices to show $k_{X}=k_{Y}$. If $k_{Y} \subsetneq k_{X}$, then $W\left(k_{Y}\right) \otimes_{\mathbb{Z}} \mathbb{Q} \subsetneq W\left(k_{X}\right) \otimes_{\mathbb{Z}} \mathbb{Q}$. This means that the induced map

$$
W\left(k_{Y}\right) \otimes_{\mathbb{Z}} \mathbb{Q}=H^{0}\left(Y, W \mathcal{O}_{Y}\right) \otimes_{\mathbb{Z}} \mathbb{Q} \rightarrow H^{0}\left(X, W \mathcal{O}_{X}\right) \otimes_{\mathbb{Z}} \mathbb{Q}=W\left(k_{X}\right) \otimes_{\mathbb{Z}} \mathbb{Q}
$$

is not surjective, which leads to a contradiction.

Lemma 2.25. Let $f: X \rightarrow Y$ be a continuous map between topological spaces. Let $A$ be a sheaf of abelian groups on $X$. Assume that there exists a finite subset $\left\{x_{1}, \cdots, x_{r}\right\} \subset X$ which satisfies the following properties.

(1) For every $1 \leq j \leq r, x_{j}$ is a closed point, that is, $\left\{x_{j}\right\}$ is a closed subset of $X$.

(2) For every $1 \leq j \leq r, f\left(x_{j}\right)$ is a closed point, that is, $\left\{f\left(x_{j}\right)\right\}$ is a closed subset of $Y$.

(3) $\left.A\right|_{X \backslash\left\{x_{1}, \cdots, x_{r}\right\}}=0$.

Then, $R^{p} f_{*} A=0$ for every $p>0$.

Proof. Since the condition (3) implies $i_{*} i^{-1} A \cong A$ where $i:\left\{x_{1}, \cdots, x_{r}\right\} \hookrightarrow$ $X$, we can assume $X=\left\{x_{1}, \cdots, x_{r}\right\}$. By the condition (2), we can also assume that $f(X)$ is one point ( $\operatorname{set}\{y\}:=f(X))$. Then, by the condition (1), for every sheaf of abelian groups $B$ on $X$ and every open subset $y \in Y^{\prime} \subset Y$, we see

$$
\left(f_{*} B\right)\left(Y^{\prime}\right)=B\left(f^{-1}\left(Y^{\prime}\right)\right)=B_{x_{1}} \oplus \cdots \oplus B_{x_{r}} .
$$

Thus, the functor $f_{*}$ is exact and hence $R^{p} f_{*} A=0$ for every $p>0$. 


\section{WO-RATIONALiTy OF KLT THREEFOLDS}

For a variety $X$ admitting a resolution of singularities, we consider the following condition:

(a) There exists a proper birational morphism $\varphi: V \rightarrow X$ from a smooth variety $V$ such that $R^{i} \varphi_{*}\left(W \mathcal{O}_{V, \mathbb{Q}}\right)=0$ holds for every $i>0$.

A variety satisfying this property is said to have $W \mathcal{O}$-rational singularities (see [CR12, Corollary 4.5.1] and Remark 2.17). This property is stronger than the definition of Witt-rational singularity by Blickle and Esnault [BE08, and weaker than that of the usual rational singularity (see CR12, Proposition 4.4.17]). The original $W \mathcal{O}$-rational singularity was defined by Chatzistamatiou and Rülling and studied in [CR12. For instance, it is known that the condition (a) is independent of the choice of a resolution:

Remark 3.1. When $X$ satisfies the condition (a), it is known that

$$
R^{i} \varphi_{*}\left(W \mathcal{O}_{V, \mathbb{Q}}\right)=0
$$

holds for every $i>0$ and for any resolution $\varphi$. More generally, it is known that the condition (a) is equivalent to the condition $R^{i} \varphi_{*}\left(W \mathcal{O}_{V, \mathbb{Q}}\right)=0$ holds for every $i>0$ for some (or any) quasi-resolution $\varphi: V \rightarrow X$ (See [CR12, Corollary 4.5.1]).

We note that the existence of the quasi-resolution is known for varieties in any dimension by [dJ97, Corollary 5.15], and the $W \mathcal{O}$-rationality is defined using quasi-resolutions when we do not assume the existence of resolutions. We also note that for a proper birational morphism $\varphi: V \rightarrow X$ between smooth varieties the vanishing of the higher direct image sheaf $R^{i} \varphi_{*} \mathcal{O}_{X}$ for $i>0$ is known by [CR11, Theorem 2].

In this section, we show that the property (a) is preserved under plcontractions and pl-flips in dimension three (Subsection 3.1). As a consequence, we see that an arbitrary klt threefold, of characteristic $p>5$, has $W \mathcal{O}$-rational singularities (Theorem 3.16). For a characteristic-independent argument, we also discuss the bijectivity of the normalization of plt centers in Subsection 3.2. As a consequence in characteristic $p \leq 5$, we reduce the problem on the existence of flips to a certain special cases (Theorem 3.15). The readers who are not interested in the low characteristic case may skip Subsection 3.2 (see also Remark 3.5).

3.1. WO-rationality under pl-contractions and pl-flips. In this subsection, we prove Proposition 3.4 and Proposition 3.8, which state that the $W \mathcal{O}$-rationality is preserved under pl-contractions and pl-flips.

First we recall the definition of pl-contractions and pl-flips.

Definition 3.2. Let $(X, \Delta)$ be a dlt pair and let $S=\sum_{i \in I} S_{i}$ be the irreducible decomposition of $S:=\llcorner\Delta\lrcorner$. We say a proper birational morphism $f: X \rightarrow Y$ is a pl-divisorial (resp. pl-flipping) contraction if the following conditions hold.

- $f_{*} \mathcal{O}_{X}=\mathcal{O}_{Y}$.

- $\rho(X / Y)=1$.

- $-\left(K_{X}+\Delta\right)$ is $f$-ample.

- $-S_{0}$ is $f$-ample for some $0 \in I$. 
- $\operatorname{dim} \operatorname{Ex}(f)=\operatorname{dim} X-1$ (resp. $\operatorname{dim} \operatorname{Ex}(f)<\operatorname{dim} X-1)$.

For a pl-flipping contraction $f: X \rightarrow Y$, a proper birational morphism $f^{+}: X^{+} \rightarrow Y$ is called a pl-flip if the following conditions hold

- $f_{*}^{+} \mathcal{O}_{X^{+}}=\mathcal{O}_{Y}$.

- $\rho\left(X^{+} / Y\right)=1$.

- $K_{X^{+}}+\Delta^{+}$is $\mathbb{Q}$-Cartier and $f^{+}$-ample, where $\Delta^{+}$is the strict transform of $\Delta$ on $X^{+}$.

- $\operatorname{dim} \operatorname{Ex}\left(f^{+}\right)<\operatorname{dim} X-1$.

The advantage of considering pl-contractions is that some problems on the threefold $X$ can be reduced to problems on the surfaces $S_{i}$. The following result on surfaces plays a crucial role in the proof of Proposition 3.4.

Proposition 3.3. Let $\left(S, \Delta_{S}\right)$ be a dlt surface over a perfect field $k$ of characteristic $p>0$ and let $f: S \rightarrow U$ be a projective morphism to a separated scheme $U$ of finite type over $k$. If $-\left(K_{S}+\Delta_{S}\right)$ is $f$-ample, then $R^{i} f_{*} \mathcal{O}_{S}=0$ holds for all $i>0$. In particular, $R^{i} f_{*}\left(W \mathcal{O}_{S, \mathbb{Q}}\right)=0$ holds for every $i>0$.

Proof. By Lemma 2.19 and Lemma 2.21, it suffices to show that $R^{i} f_{*} \mathcal{O}_{S}=0$ for $i>0$. Since the base change $\left(X \times_{k} \bar{k}, \Delta \times_{k} \bar{k}\right)$ to the algebraic closure is also dlt, we may assume that $k$ is an algebraically closed field. Since dlt surfaces are always $\mathbb{Q}$-factorial (cf. [FT12, Proposition 6.3]), we may assume that $\left(S, \Delta_{S}\right)$ is klt. We may also assume that $f_{*} \mathcal{O}_{S}=\mathcal{O}_{U}$ by the Stein factorization.

If $\operatorname{dim} U \geq 1$, then the assertion follows from [Tan15, Theorem 2.12]. Suppose $\operatorname{dim} U=0$. In this case, the assertion follows from the fact that $S$ is a rational surface ([Tan15, Theorem 3.5]) with rational singularities (cf. [FT12, Theorem 6.4]).

We prove that the $W \mathcal{O}$-rationality is preserved under pl-contractions.

Proposition 3.4. Let $k$ be a perfect field of characteristic $p>0$. Let $(X, \Delta)$ be a dlt threefold over $k$ and let $S=\sum_{i=0}^{r} S_{i}$ be the irreducible decomposition of $S:=\llcorner\Delta\lrcorner$. Let $f: X \rightarrow Y$ be a projective birational morphism with the following properties.

- $f_{*} \mathcal{O}_{X}=\mathcal{O}_{Y}$.

- $-\left(K_{X}+\Delta\right)$ is $f$-ample.

- $-S_{0}$ is $f$-ample.

- The normalization $\nu: S_{0}^{N} \rightarrow S_{0}$ is a universal homeomorphism.

Then, the following assertions hold.

(1) $R^{i} f_{*}\left(W \mathcal{O}_{X, \mathbb{Q}}\right)=0$ for every $i>0$.

(2) If $X$ has $W \mathcal{O}$-rational singularities, then so does $Y$.

Remark 3.5. The fourth assumption is known to be true when $(X, \Delta)$ is $\mathbb{Q}$-factorial dlt threefold of characteristic $p>5$. Indeed, in this case, $S_{0}$ is known to be normal [HX15, Proposition 4.1] (cf. Theorem 2.11). In the next subsection, this condition is called "the condition (b)" and we study its behavior under pl-contractions and pl-flips in arbitrary positive characteristic. 
Proof of Proposition 3.4. The assertion (2) follows from (1) by the Grothendieck spectral sequence. In what follows, we prove the assertion (1). Since $-S_{0}$ is $f$-ample, we see $\operatorname{Ex}(f) \subset S_{0}$. Therefore, by Proposition 2.23 , it follows that

$$
R^{i} f_{*}\left(W I_{S_{0}, \mathbb{Q}}\right)=0
$$

for any $i>0$, where $I_{S_{0}} \subset \mathcal{O}_{X}$ is the defining ideal sheaf of $S_{0} \subset X$. By the following exact sequence:

$$
0 \rightarrow W I_{S_{0}, \mathbb{Q}} \rightarrow W \mathcal{O}_{X, \mathbb{Q}} \rightarrow \iota_{*} W \mathcal{O}_{S_{0}, \mathbb{Q}} \rightarrow 0,
$$

where $\iota: S_{0} \hookrightarrow X$, it follows that

$$
R^{i} f_{*}\left(W \mathcal{O}_{X, \mathbb{Q}}\right) \cong R^{i} f_{*}\left(\iota_{*} W \mathcal{O}_{S_{0}, \mathbb{Q}}\right)
$$

for $i>0$. Since $\nu: S_{0}^{N} \rightarrow S_{0}$ is a universal homeomorphism, it follows that $\nu_{*} W \mathcal{O}_{S_{0}^{N}} \cong W \mathcal{O}_{S_{0}, \mathbb{Q}}$ by Lemma 2.22, and we obtain

$$
R^{i} f_{*}\left(\iota_{*} W \mathcal{O}_{S_{0}, \mathbb{Q}}\right) \cong R^{i} f_{*}\left(\iota_{*} \nu_{*} W \mathcal{O}_{S_{0}^{N}, \mathbb{Q}}\right) \cong R^{i}(f \circ \iota \circ \nu)_{*}\left(W \mathcal{O}_{S_{0}^{N}, \mathbb{Q}}\right) .
$$

Note that the latter isomorphism follows from $R^{i}(\iota \circ \nu)_{*}\left(W \mathcal{O}_{S_{0}^{N}, \mathbb{Q}}\right)=0$ for $i>0$ by Lemma 2.20. Since the pair $\left(S_{0}^{N}, \Delta_{S_{0}^{N}}\right)$, defined by $\left.\left(K_{X}+\Delta\right)\right|_{S_{0}^{N}}=$ $K_{S_{0}^{N}}+\Delta_{S_{0}^{N}}$, is dlt by adjunction, the last sheaf is zero by Proposition 3.3 , which completes the proof.

Next, we show Proposition 3.8. We start with the following result on surfaces over a (possibly imperfect) field (see Remark 2.1).

Lemma 3.6. Let $f: T \rightarrow S$ be a proper birational morphism of twodimensional excellent schemes, where $T$ is regular and $\left(S, \Delta_{S}\right)$ is dlt for some effective $\mathbb{Q}$-divisor $\Delta_{S}$ on $S$. Then, $R^{i} f_{*} \mathcal{O}_{T}=0$ holds for $i>0$.

Proof. We may replace $f$ with the minimal resolution of $S$. Note that the minimal resolutions exist by [Kol13, Theorem 10.5]. Then, the $\mathbb{Q}$-divisor $\Delta_{T}$, defined by $K_{T}+\Delta_{T}=f^{*}\left(K_{S}+\Delta_{S}\right)$, is effective by the negativity lemma. If $E$ is an $f$-exceptional curve on $T$, then the image $f(E)$ is contained in the non-regular locus of $S$, and hence $a_{E}\left(S, \Delta_{S}\right)<0$. Since $\left(S, \Delta_{S}\right)$ is dlt, we obtain $a_{E}\left(S, \Delta_{S}\right)>-1$. This means that, if $f$ is not an isomorphism, then some coefficient of $\Delta_{T}$ is less than one. Therefore, $R^{1} f_{*} \mathcal{O}_{T}=0$ follows from [Kol13, Theorem 10.4] by letting

$$
L:=\mathcal{O}_{T}, \quad N:=(m-1)\left(K_{T}+\Delta_{T}\right)-f^{*}\left(m\left(K_{S}+\Delta_{S}\right)\right),
$$

where $m$ is a positive integer such that $m\left(K_{S}+\Delta_{S}\right)$ is Cartier.

Lemma 3.6 provides the following consequence on threefolds.

Lemma 3.7. Let $(X, \Delta)$ be a dlt threefold over a perfect field $k$ of characteristic $p>0$. Let $\varphi: V \rightarrow X$ be a proper birational morphism from a smooth threefold $V$. Then, the following assertions hold.

(1) There exists a zero-dimensional closed subset $Z$ of $X$ such that

$$
\left.\left(R^{i} \varphi_{*} \mathcal{O}_{V}\right)\right|_{X \backslash Z}=0
$$

for any $i>0$. 
(2) There exists a zero-dimensional closed subset $Z$ of $X$ such that

$$
\left.\left(R^{i} \varphi_{*}\left(W \mathcal{O}_{V}\right)\right)\right|_{X \backslash Z}=0
$$

for any $i>0$.

Proof. The assertion (2) follows from (1) by Lemma 2.19, In what follows, we prove the assertion (1). Suppose that $\operatorname{Supp}\left(R^{i} \varphi_{*} \mathcal{O}_{V}\right)$ contains a curve $C$ for some $i>0$ and we derive a contradiction. Let $\xi_{C}$ be the generic point of $C$ and consider the following square of a fiber product:

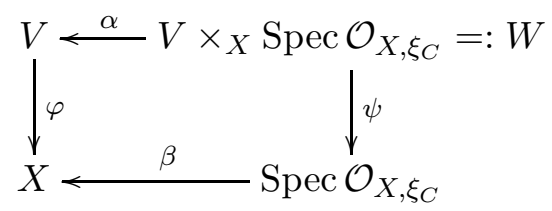

By the flat base change theorem, it follows that

$$
0 \neq \beta^{*} R^{i} \varphi_{*} \mathcal{O}_{V} \cong R^{i} \psi_{*}\left(\alpha^{*} \mathcal{O}_{V}\right) \cong R^{i} \psi_{*} \mathcal{O}_{W} .
$$

Note that $\left(\operatorname{Spec} \mathcal{O}_{X, \xi_{C}},\left.\Delta\right|_{\operatorname{Spec}} \mathcal{O}_{X, \xi_{C}}\right)$ is a two dimensional excellent scheme and that $W$ is regular. Hence, we obtain a contradiction by Lemma 3.6 .

The $W \mathcal{O}$-rationality is preserved under pl-flips.

Proposition 3.8. Let $k$ be a perfect field of characteristic $p>0$, and let $g: Y \rightarrow Z$ be a proper birational morphism between normal threefolds over $k$ such that any fiber of $g$ is at most one dimensional. Assume that $\left(Y, \Delta_{Y}\right)$ is dlt for some effective $\mathbb{Q}$-divisor $\Delta_{Y}$. If $Z$ has $W \mathcal{O}$-rational singularities, then so does $Y$.

Proof. Let $\psi: V \rightarrow Y$ be a resolution of $Y$ (cf. [CP08]). We prove $R^{i} \psi_{*}\left(W \mathcal{O}_{V, \mathbb{Q}}\right)=$ 0 for $i>0$. We denote by $\varphi$ the composition $g \circ \psi$ :

$$
\varphi: V \stackrel{\psi}{\rightarrow} Y \stackrel{g}{\rightarrow} Z
$$

Consider the Grothendieck spectral sequence:

$$
E_{2}^{i, j}=R^{i} g_{*} R^{j} \psi_{*}\left(W \mathcal{O}_{V, \mathbb{Q}}\right) \Longrightarrow E^{i+j}=R^{i+j} \varphi_{*}\left(W \mathcal{O}_{V, \mathbb{Q}}\right) .
$$

Here, we claim that $E_{2}^{i, j}=0$ holds for $i>0$.

Claim 3.9. $E_{2}^{i, j}=R^{i} g_{*} R^{j} \psi_{*}\left(W \mathcal{O}_{V, \mathbb{Q}}\right)=0$ holds for every $i>0$ and $j \geq 0$.

First we assume this claim and finish the proof. This claim and the spectral sequence above yield

$$
g_{*}\left(R^{j} \psi_{*}\left(W \mathcal{O}_{V, \mathbb{Q}}\right)\right) \cong R^{j} \varphi_{*}\left(W \mathcal{O}_{V, \mathbb{Q}}\right),
$$

and this is zero for $j>0$ by the $W \mathcal{O}$-rationality of $Z$ (cf. Remark 3.1). On the other hand, by Lemma 3.7, the support of $R^{j} \psi_{*}\left(W \mathcal{O}_{V}\right)$ is at most a finite set for $j>0$. Therefore, $g_{*} R^{j} \psi_{*}\left(W \mathcal{O}_{V, \mathbb{Q}}\right)=0$ implies $R^{j} \psi_{*}\left(W \mathcal{O}_{V, \mathbb{Q}}\right)=0$, which shows the $W \mathcal{O}$-rationality of $Y$.

Proof of Claim 3.9. We divide the proof into three cases: (I) $i>0, j>0$, (II) $i=1, j=0$, and (III) $i \geq 2, j=0$.

(I) Suppose that $i>0$ and $j>0$. By Lemma 3.7. $\operatorname{Supp}\left(R^{j} \psi_{*}\left(W \mathcal{O}_{V}\right)\right)$ is at most a finite set. Therefore, $R^{i} g_{*} R^{j} \psi_{*}\left(W \mathcal{O}_{V}\right)=0$ holds by Lemma 2.25, In particular, we get $R^{i} g_{*} R^{j} \psi_{*}\left(W \mathcal{O}_{V, \mathbb{Q}}\right)=0$. 
(II) Suppose that $i=1$ and $j=0$. Then $E_{2}^{1,0}=0$ follows from the injectivity $E_{2}^{1,0} \hookrightarrow E^{1}$ and the vanishing $E^{1}=R^{1} \varphi_{*}\left(W \mathcal{O}_{V, \mathbb{Q}}\right)=0$ which follows from the $W \mathcal{O}$-rationality of $Z$.

(III) Suppose that $i \geq 2$ and $j=0$. Then by Lemma 2.20,

$$
E_{2}^{i, 0}=R^{i} g_{*}\left(\psi_{*}\left(W \mathcal{O}_{V, \mathbb{Q}}\right)\right)=R^{i} g_{*}\left(W \mathcal{O}_{Y, \mathbb{Q}}\right)
$$

turns out to be zero since any fiber of $g$ is at most one dimensional.

This completes the proof of Proposition 3.8 .

3.2. Normalization for plt centers under pl-contractions and plflips. For a $\mathbb{Q}$-factorial dlt threefold $(Y, \Delta)$, we consider the following condition:

(b) The normalization of each irreducible component of $\llcorner\Delta\lrcorner$ is a universal homeomorphism.

In this subsection, we study its behavior under pl-contractions (Proposition 3.11) and pl-flips (Proposition 3.12).

First we prove the following lemma, which is a consequence of the KollárShokurov connectedness lemma for surfaces ([Tan16, Proposition 2.3]).

Lemma 3.10. Let $\left(S, \Delta_{S}\right)$ be a dlt surface over a perfect field of characteristic $p>0$ and let $\pi: S \rightarrow U$ be a projective surjective morphism such that every fiber of $\pi$ is geometrically connected. Assume that $-\left(K_{S}+\Delta_{S}\right)$ is $\pi$-ample. Let $0 \leq D \leq\left\llcorner\Delta_{S}\right\lrcorner$ be a divisor satisfying $D=\llcorner D\lrcorner$. Then, $\operatorname{Supp}(D) \cap \pi^{-1}(u)$ is geometrically connected for every closed point $u \in U$.

Proof. Replacing $k$ by the algebraic closure, we may assume that $k$ is algebraically closed. Taking the Stein factorization of $\pi$, we can assume that $\pi_{*} \mathcal{O}_{S}=\mathcal{O}_{U}$. Note that $S$ is $\mathbb{Q}$-factorial (cf. [FT12, Proposition 6.3]). By taking a small perturbation, we can find $0 \leq \Delta_{S}^{\prime} \leq \Delta_{S}$ such that $D=\left\llcorner\Delta_{S}^{\prime}\right\lrcorner$ and that $-\left(K_{S}+\Delta_{S}^{\prime}\right)$ is $\pi$-ample. Then, the assertion follows from [Tan16, Proposition 2.2].

The condition (b) is preserved under pl-contractions.

Proposition 3.11. Let $k$ be a perfect field of characteristic $p>0$. Let $(X, \Delta)$ be a $\mathbb{Q}$-factorial dlt threefold over $k$ and let $S=\sum_{i=0}^{r} S_{i}$ be the irreducible decomposition of $S:=\llcorner\Delta\lrcorner$. Let $f: X \rightarrow Y$ be a projective birational morphism with the following properties.

- $f_{*} \mathcal{O}_{X}=\mathcal{O}_{Y}$.

- $-\left(K_{X}+\Delta\right)$ is $f$-ample.

- $-S_{0}$ is f-ample.

- For every $i$, the normalization morphism $S_{i}^{N} \rightarrow S_{i}$ is a universal homeomorphism.

Let $T_{i}:=f\left(S_{i}\right)$ be the scheme-theoretic image. Then for every $i \in I$, the normalization morphism $T_{i}^{N} \rightarrow T_{i}$ is a universal homeomorphism.

Proof. For each $i$, it is sufficient to show that every fiber of $g_{i}:=\left.f\right|_{S_{i}}: S_{i} \rightarrow$ $T_{i}$ is geometrically connected.

Suppose $i=0$. Since $-S_{0}$ is $f$-ample, we see $\operatorname{Ex}(f) \subset S_{0}$. As $f$ has geometrically connected fibers, every fiber of $g_{0}: S_{0} \rightarrow T_{0}$ is also geometrically connected. 
Suppose $i \neq 0$. Let $y \in T_{i} \subset Y$. It is enough to show that $g_{i}^{-1}(y)$ is geometrically connected. We can assume that $y \in f(\operatorname{Ex}(f))$, in particular, $y \in T_{0}$. It follows that

$$
g_{i}^{-1}(y)=S_{0} \cap\left(\left.f\right|_{S_{i}}\right)^{-1}(y)=S_{0} \cap S_{i} \cap f^{-1}(y)=S_{i} \cap g_{0}^{-1}(y) .
$$

Hence it is sufficient to show that each fiber of the composition

$$
C_{i}:=S_{0} \cap S_{i} \hookrightarrow S_{0} \stackrel{g_{0}}{\longrightarrow} T_{0}
$$

is either empty or geometrically connected. Now, we set $\nu_{0}: S_{0}^{N} \rightarrow S_{0}$ and define $\Delta_{S_{0}^{N}}$ by $\left.\left(K_{X}+\Delta\right)\right|_{S_{0}^{N}}=K_{S_{0}^{N}}+\Delta_{S_{0}^{N}}$. Note that the intersection $C_{i}:=$ $S_{0} \cap S_{i}$ is either empty or purely one-dimensional because $X$ is $\mathbb{Q}$-factorial. Thus, $\nu_{0}^{-1}\left(C_{i}\right)$ is also purely one-dimensional and $\nu_{0}^{-1}\left(C_{i}\right) \subset \operatorname{Supp}\left(\left\llcorner\Delta_{S_{0}^{N}}\right\lrcorner\right)$ holds by adjunction. Further, $\left(S_{0}^{N}, \Delta_{S_{0}^{N}}\right)$ is dlt and $-\left(K_{S_{0}^{N}}+\Delta_{S_{0}^{N}}\right)$ is $\left(g_{0} \circ \nu\right)$ ample. Then, by Lemma 3.10, it follows that each fiber of the composition

$$
\nu_{0}^{-1}\left(C_{i}\right) \hookrightarrow S_{0}^{N} \stackrel{g_{0} \circ \nu}{\longrightarrow} T_{0}
$$

is geometrically connected, which is what we want to show.

Condition (b) is preserved under pl-flips if we make an additional assumption on the Witt vector cohomology.

Proposition 3.12. Let $g: Y \rightarrow Z$ be a proper birational morphism between normal threefolds over a perfect field of characteristic $p>0$, such that any fiber of $g$ is at most one dimensional. Let $S_{Y}$ be a prime divisor on $Y$ and set $S_{Z}:=g_{*}\left(S_{Y}\right)$. Assume the following three conditions.

- $S_{Y}$ is regular in codimension one.

- The normalization $S_{Z}^{N} \rightarrow S_{Z}$ is a universal homeomorphism.

- $R^{1} g_{*}\left(W \mathcal{O}_{Y, \mathbb{Q}}\right)=0$.

Then the normalization $\nu: S_{Y}^{N} \rightarrow S_{Y}$ is a universal homeomorphism.

Proof. Set $\left.g\right|_{S_{Y}}=: h: S_{Y} \rightarrow S_{Z}$. First we prove $R^{1} h_{*}\left(W \mathcal{O}_{S_{Y}, \mathbb{Q}}\right)=0$. Consider the following exact sequence:

$$
0 \rightarrow W I_{S_{Y}, \mathbb{Q}} \rightarrow W \mathcal{O}_{Y, \mathbb{Q}} \rightarrow W \mathcal{O}_{S_{Y}, \mathbb{Q}} \rightarrow 0 .
$$

Since $\operatorname{dim} \operatorname{Ex}(g) \leq 1$, it follows that $R^{2} g_{*}\left(W I_{S_{Y}, \mathbb{Q}}\right)=0$ by Lemma 2.20 . Thus, $R^{1} h_{*}\left(W \mathcal{O}_{S_{Y}, \mathbb{Q}}\right)=0$ follows from the assumption $R^{1} g_{*}\left(W \mathcal{O}_{Y, \mathbb{Q}}\right)=0$.

Let $C \subset S_{Y}$ be the conductor of the normalization $\nu: S_{Y}^{N} \rightarrow S_{Y}$ and let $D:=\nu^{-1}(C) \subset S_{Y}^{N}$ be its scheme-theoretic inverse image. Then we have the following exact sequence:

$$
0 \rightarrow W \mathcal{O}_{S_{Y}, \mathbb{Q}} \rightarrow \nu_{*} W \mathcal{O}_{S_{Y}^{N}, \mathbb{Q}} \oplus W \mathcal{O}_{C, \mathbb{Q}} \rightarrow \nu_{*} W \mathcal{O}_{D, \mathbb{Q}} \rightarrow 0 .
$$

Since $R^{1} h_{*}\left(W \mathcal{O}_{S_{Y}, \mathbb{Q}}\right)=0$, we obtain the exact sequence

$$
0 \rightarrow h_{*} W \mathcal{O}_{S_{Y}, \mathbb{Q}} \rightarrow h_{*} \nu_{*} W \mathcal{O}_{S_{Y}^{N}, \mathbb{Q}} \oplus h_{*} W \mathcal{O}_{C, \mathbb{Q}} \rightarrow h_{*} \nu_{*} W \mathcal{O}_{D, \mathbb{Q}} \rightarrow 0 .
$$

Note that $S_{Y}^{N} \rightarrow S_{Z}^{N}$ is a birational morphism with geometrically connected fibers by the Zariski main theorem. Since the normalization $S_{Z}^{N} \rightarrow S_{Z}$ is a universal homeomorphism, both of $h \circ \nu: S_{Y}^{N} \rightarrow S_{Z}$ and $h$ have geometrically connected fibers, hence we obtain

$$
h_{*} W \mathcal{O}_{S_{Y}, \mathbb{Q}} \cong W \mathcal{O}_{S_{Z}, \mathbb{Q}} \cong h_{*} \nu_{*} W \mathcal{O}_{S_{Y}^{N}, \mathbb{Q}}
$$


by Lemma 2.22. Thus, we obtain $h_{*} W \mathcal{O}_{C, \mathbb{Q}} \cong h_{*} \nu_{*} W \mathcal{O}_{D, \mathbb{Q}}$ by the exact sequence above, and hence

$$
H^{0}\left(C, W \mathcal{O}_{C, \mathbb{Q}}\right) \cong H^{0}\left(D, W \mathcal{O}_{D, \mathbb{Q}}\right)
$$

Note that $C$ and $D$ are zero-dimensional by the first assumption. Therefore, by Lemma 2.24, it follows that $C \rightarrow D$ is a universal homeomorphism, hence so is $\nu: S_{Y}^{N} \rightarrow S_{Y}$.

3.3. WO-rationality of klt threefolds. In this subsection, we prove that the conditions (a) and (b) hold for $\mathbb{Q}$-factorial dlt threefolds modulo the following conjecture on the existence of flips.

Conjecture 3.13. Let $k$ be a perfect field of characteristic $p>0$. Let $f:(Y, T+B) \rightarrow Z$ be a pl-flipping contraction from a $\mathbb{Q}$-factorial plt threefold $(Y, T+B)$ over $k$, where $\llcorner T+B\lrcorner=T$. If the normalization of $T$ is a universal homeomorphism, then a flip of $f$ exists.

We note that Conjecture 3.13 is known for $p>5$. More generally, the existence of flips is known for $\mathbb{Q}$-factorial dlt threefolds over a perfect field of characteristic $p>5$ (cf. Theorem 2.10).

Theorem 3.14. Fix a perfect field $k$ of characteristic $p>0$ and assume that Conjecture 3.13 holds for $k$.

Let $(X, \Delta)$ be a $\mathbb{Q}$-factorial dlt threefold over $k$. Then the following assertions hold.

(1) X has WO-rational singularities.

(2) For any irreducible component $S$ of $\llcorner\Delta\lrcorner$, its normalization morphism $\nu: S^{N} \rightarrow S$ is a universal homeomorphism.

Proof. Since $X$ is $\mathbb{Q}$-factorial, we may assume that $\Delta$ is a prime divisor for the proof of (2), and $\Delta=0$ for (1). Set $S:=\Delta$.

Let $f: Y \rightarrow X$ be a $\log$ resolution of $(X, S)$. Then we may write

$$
K_{Y}+S_{Y}+E=f^{*}\left(K_{X}+S\right)+E^{\prime} \sim_{\mathbb{Q}, X} E^{\prime},
$$

where $S_{Y}$ is the strict transform of $S, E$ is the sum of all the $f$-exceptional prime divisors, and $E^{\prime}$ is an effective $f$-exceptional $\mathbb{Q}$-divisor. Note that $\operatorname{Ex}(f)=\operatorname{Supp} E^{\prime}$ as $(X, \Delta)$ is plt. We shall inductively construct a $\left(K_{Y}+\right.$ $\left.S_{Y}+E\right)$-MMP over $X$

$$
\left(Y, S_{Y}+E\right)=:\left(Y_{0}, S_{0}+E_{0}\right) \stackrel{g_{0}}{\rightarrow}\left(Y_{1}, S_{1}+E_{1}\right)_{\stackrel{-}{g_{1}} \rightarrow}^{\rightarrow} \cdots,
$$

with the following two conditions:

(a) ${ }_{j} Y_{j}$ has $W \mathcal{O}$-rational singularities.

(b) ${ }_{j}$ The normalization of each irreducible component of $S_{j}+E_{j}$ is a universal homeomorphism.

Further, we shall prove that this MMP terminates and ends with $X$ itself. We note that the conditions $(\mathrm{a})_{0}$ and $(\mathrm{b})_{0}$ are satisfied since $\left(Y_{0}, S_{0}+E_{0}\right)$ is $\log$ smooth.

Suppose that we have already constructed $\left(Y_{j}, S_{j}+E_{j}\right)$ with the conditions (a) ${ }_{j}$ and (b) $)_{j}$. If $K_{Y_{j}}+S_{j}+E_{j}$ is nef over $X$, then $Y_{j}=X$ holds by the negativity lemma and the $\mathbb{Q}$-factoriality of $X$. Suppose that $K_{Y_{j}}+S_{j}+E_{j}$ is not nef over $X$. Since $K_{Y_{j}}+S_{j}+E_{j}$ is pseudo-effective over $X$, by 
Lemma 2.2. we can find a $\left(K_{Y_{j}}+S_{j}+E_{j}\right)$-negative extremal ray $R$ of $\overline{N E}\left(Y_{i} / X\right)$. Since the condition (b) ${ }_{j}$ holds and $F \cdot R<0$ holds for some component $F$ of $E_{j}$, the assumptions in Lemma 2.4 are satisfied. Hence, we obtain a projective birational morphism $h: Y_{j} \rightarrow Z$ over $X$ corresponding to $R$. Then Proposition 3.4 and Proposition 3.11 ensure that the new pair $\left(Z, h_{*} S_{j}+h_{*} E_{j}\right)$ satisfies the conditions (a) and (b). Hence, if $h$ is a divisorial contraction, it is sufficient to set

$$
Y_{j+1}:=Z, \quad S_{j+1}:=h_{*} S_{j}, \quad E_{j+1}:=h_{*} E_{j}
$$

for our purpose. Suppose that $h$ is a flipping contraction. Since $\left(Y_{j}, S_{j}+E_{j}\right)$ satisfies the condition (b) ${ }_{j}$, we may apply Conjecture 3.13, and a flip $h^{+}$: $Y_{j+1} \rightarrow Z$ of $h$ exists. Set $S_{j+1}$ and $E_{j+1}$ to be the proper transforms on $Y_{j+1}$ of $S_{j}$ and $E_{j}$, respectively. Then, the new pair $\left(Y_{j+1}, S_{j+1}+E_{j+1}\right)$ satisfies the condition $(\mathrm{a})_{j+1}$ by Proposition 3.8, Further, by Lemma 2.5, each irreducible component of $S_{j+1}+E_{j+1}$ is regular in codimension one. Thus we may apply Proposition 3.12 and the condition $(\mathrm{b})_{j+1}$ is also satisfied.

We note that this MMP terminates by the special termination (Theorem 2.6). Since this MMP ends with $X$, the assertions (1) and (2) follow from the conditions $(\mathrm{a})_{j}$ and $(\mathrm{b})_{j}$ respectively.

In characteristic $p \leq 5$, we obtain the following reduction result by the same proof as in [Fuj07, Theorem 4.3.7].

Theorem 3.15. If Conjecture 3.13 holds, then a flip of every flipping contraction exists. In other words, when we show the existence of pl-flips for $\mathbb{Q}$-factorial plt threefolds $(X, S+B)$ with $\llcorner S+B\lrcorner=S$, we may assume that the normalization of $S$ is a universal homeomorphism.

In characteristic $p>5$, any klt threefold (which is not necessarily $\mathbb{Q}$ factorial) has $W \mathcal{O}$-rational singularities.

Theorem 3.16. Let $(X, \Delta)$ be a klt threefold over a perfect field of characteristic $p>5$. Then $X$ has $W \mathcal{O}$-rational singularities.

Proof. The assertion is proved in Theorem 3.14 when $X$ is $\mathbb{Q}$-factorial. Thus it suffices to find a proper birational morphism $g: Y \rightarrow X$ from a $\mathbb{Q}$-factorial klt threefold satisfying $R^{i} g_{*} W \mathcal{O}_{Y, \mathbb{Q}}=0$ for $i>0$. Since the problem is local and the non- $\mathbb{Q}$-factorial points on $X$ are isolated, we may assume that $X$ contains the unique non- $\mathbb{Q}$-factorial point $z$.

By Proposition 2.15, we can find a projective birational morphism $h$ : $Y \rightarrow X$ with the following conditions:

- $Y$ is $\mathbb{Q}$-factorial and klt,

- $h$ is isomorphic over $X \backslash\{z\}$.

- $E:=h^{-1}(z)_{\text {red }}$ is a surface of Fano type.

By the following short exact sequence

$$
0 \rightarrow W I_{E, \mathbb{Q}} \rightarrow W \mathcal{O}_{Y, \mathbb{Q}} \rightarrow W \mathcal{O}_{E, \mathbb{Q}} \rightarrow 0
$$

and the vanishing $R^{i} h_{*}\left(W I_{E, \mathbb{Q}}\right)=0$ for $i>0$ (Proposition 2.23), we obtain

$$
R^{i} h_{*}\left(W \mathcal{O}_{Y, \mathbb{Q}}\right) \cong R^{i} h_{*}\left(W \mathcal{O}_{E, \mathbb{Q}}\right)
$$

for $i>0$. The latter sheaf $R^{i} h_{*}\left(W \mathcal{O}_{E, \mathbb{Q}}\right)$ is zero by Proposition 3.3 , 
3.4. Examples. In this subsection, we give two examples on rational and $W \mathcal{O}$-rational singularities. Before we construct the first example, we show an auxiliary lemma.

Lemma 3.17. Let $f: X \rightarrow Y$ be a proper birational morphism of normal surfaces over an algebraically closed field of characteristic $p>0$. Assume that $E:=\operatorname{Ex}(f)$ is a rational curve whose normalization is a universal homeomorphism. Then $R^{i} f_{*}\left(W \mathcal{O}_{X, \mathbb{Q}}\right)=0$ for $i>0$.

Proof. By Proposition 2.23, we obtain

$$
R^{i} f_{*}\left(W \mathcal{O}_{X, \mathbb{Q}}\right) \cong H^{i}\left(E, W \mathcal{O}_{E, \mathbb{Q}}\right) .
$$

Let $\nu: \mathbb{P}^{1} \rightarrow E$ be the normalization. It follows from Lemma 2.22 that $\nu_{*} W \mathcal{O}_{\mathbb{P}^{1}, \mathbb{Q}}=W \mathcal{O}_{E, \mathbb{Q}}$. Therefore, by Lemma 2.19 and Lemma 2.21, we obtain

for $i>0$.

$$
H^{i}\left(E, W \mathcal{O}_{E, \mathbb{Q}}\right) \cong H^{i}\left(\mathbb{P}^{1}, W \mathcal{O}_{\mathbb{P}^{1}, \mathbb{Q}}\right)=0
$$

The first example is a two-dimensional $W \mathcal{O}$-rational singularity which is not a rational singularity (cf. [CR12, Section 4.6]).

Example 3.18. Let $f: X \rightarrow Y$ be a proper birational morphism from a smooth surface to a normal surface over an algebraically closed field of characteristic $p>0$ such that $\operatorname{Ex}(f)$ is an irreducible cuspidal cubic curve. Then, the singularity of $Y$ is $W \mathcal{O}$-rational by Lemma 3.17, but not a rational singularity.

Next, we give an example which is klt but with non-rational singularities. We give an example in characteristic two. It is based on an example of weak del Pezzo surfaces $X$ with $H^{1}\left(X, \mathcal{O}_{X}\right) \neq 0$ which was given by Schröer (Theorem 8.1, 8.2 and Section 9 in [Sch07]. See also [Mad]).

Proposition 3.19. The following assertions hold.

(1) For an arbitrary imperfect field $k$ of characteristic two, there exists a three-dimensional klt pair $\left(Z, \Delta_{Z}\right)$ over $k$ such that $R^{1} g_{*} \mathcal{O}_{Y} \neq 0$ for a resolution $g: Y \rightarrow Z$ and $Z$ is the cone of some log canonical pair $(X, \Delta)$.

(2) For an arbitrary perfect field $k$ of characteristic two, there exists a four-dimensional klt pair $\left(Z^{\prime}, \Delta_{Z}^{\prime}\right)$ over $k$ such that $R^{1} g_{*}^{\prime} \mathcal{O}_{Y^{\prime}} \neq 0$ for a resolution $g^{\prime}: Y^{\prime} \rightarrow Z^{\prime}$.

Proof. Since (1) implies (2), we only show (1). Let $k$ be an arbitrary imperfect field of characteristic two. By [Sch07, Section 9], there exists a regular projective surface $X$ over $k$ such that $-K_{X}$ is nef and big and $H^{1}\left(X, \mathcal{O}_{X}\right) \neq 0$. We can find an effective $\mathbb{Q}$-divisor $\Delta$ such that $(X, \Delta)$ is klt and $-\left(K_{X}+\Delta\right)$ is ample. Fix a positive integer $\ell>0$ so that $\ell\left(K_{X}+\Delta\right)$ is Cartier and that

$$
H^{i}\left(X, \mathcal{O}_{X}\left(-m \ell\left(K_{X}+\Delta\right)\right)\right)=0
$$

for $i>0$ and $m>0$. Set $\pi: Y \rightarrow X$ to be the $\mathbb{A}^{1}$-bundle over $X$, equipped with a section $E$, such that $\left.\mathcal{O}_{Y}(E)\right|_{E} \simeq \mathcal{O}_{X}\left(\ell\left(K_{X}+\Delta\right)\right)$ and that $Y$ is an open subset of the $\mathbb{P}^{1}$-bundle $\mathbb{P}_{X}\left(\mathcal{O}_{X} \oplus \mathcal{O}_{X}\left(\ell\left(K_{X}+\Delta\right)\right)\right)$. Set $\Delta_{Y}:=\pi^{*} \Delta$. 
Then $E$ can be contracted, and we have a projective birational morphism $g: Y \rightarrow Z$ such that $\operatorname{Ex}(g)=E$ and $g(\operatorname{Ex}(g))$ is one point.

Since $\left.\left(\left(K_{Y}+\Delta_{Y}+E\right)-\frac{1}{\ell} E\right)\right|_{E} \sim_{\mathbb{Q}} 0$, we obtain $K_{Y}+\Delta_{Y}+\left(1-\frac{1}{\ell}\right) E \sim_{\mathbb{Q}} 0$. Therefore, its push-forward $g_{*}\left(K_{Y}+\Delta_{Y}+\left(1-\frac{1}{\ell}\right) E\right)=K_{Z}+\Delta_{Z}$ is $\mathbb{Q}$-Cartier, where $\Delta_{Z}:=g_{*} \Delta_{Y}$. It follows that

$$
K_{Y}+\Delta_{Y}+\left(1-\frac{1}{\ell}\right) E=g^{*}\left(K_{Z}+\Delta_{Z}\right) \text {. }
$$

Since there exists a $\log$ resolution of $(X, \Delta)$ and $Y$ is an $\mathbb{A}^{1}$-bundle over $X$, the pair $\left(Y, \Delta_{Y}+\left(1-\frac{1}{\ell}\right) E\right)$ is klt. Therefore, $\left(Z, \Delta_{Z}\right)$ is also klt.

We show $R^{1} g_{*} \mathcal{O}_{Y} \neq 0$. For this, it suffices to show that $R^{i} g_{*} \mathcal{O}_{Y}(-E)=0$ for $i>0$ since we have $H^{1}\left(X, \mathcal{O}_{X}\right) \neq 0$. We prove that $R^{i} g_{*} \mathcal{O}_{Y}(-m E)=0$ for $m>0$ by the descending induction on $m$. For $m \gg 0$, we obtain $R^{i} g_{*} \mathcal{O}_{Y}(-m E)=0$ by the Serre vanishing theorem. By an exact sequence:

$$
\left.0 \rightarrow \mathcal{O}_{Y}(-(m+1) E) \rightarrow \mathcal{O}_{Y}(-m E) \rightarrow \mathcal{O}_{Y}(-m E)\right|_{E} \rightarrow 0,
$$

it is enough to show $H^{i}\left(E,\left.\mathcal{O}_{Y}(-m E)\right|_{E}\right)=0$ for $m>0$. This holds by

$$
H^{i}\left(E,\left.\mathcal{O}_{Y}(-m E)\right|_{E}\right) \simeq H^{i}\left(X, \mathcal{O}_{X}\left(-m \ell\left(K_{X}+\Delta\right)\right)\right)=0 .
$$

Therefore, we have $R^{1} g_{*} \mathcal{O}_{Y} \neq 0$.

Remark 3.20. With the same notation as in the proof of Proposition 3.19, we can show that

$$
R^{i} g_{*}\left(W \mathcal{O}_{Y, \mathbb{Q}}\right)=0
$$

for any $i>0$. The sketch of the proof is as follows. Since $-E$ is $g$-ample, we can find $m \gg 0$ such that $R^{i} g_{*} \mathcal{O}_{Y}(-m E)=0$ for $i>0$, which implies that $R^{i} g_{*}\left(W I_{E, \mathbb{Q}}\right)=R^{i} g_{*}\left(W I_{m E, \mathbb{Q}}\right)=0$ where $I_{E}$ and $I_{m E}$ are the coherent ideal sheaves of $E$ and $m E$ respectively (cf. [BBE07, Proposition 2.1]). On the other hand, we can check that there exists a finite universal homeomorphism $X \rightarrow \mathbb{P}^{2}$ by the construction of [Sch07, Section 9]. Thus we get $H^{i}\left(E, W \mathcal{O}_{E, \mathbb{Q}}\right)=H^{i}\left(X, W \mathcal{O}_{X, \mathbb{Q}}\right)=0($ cf. Lemma 2.22) $)$. Therefore, the required vanishing $R^{i} g_{*}\left(W \mathcal{O}_{Y, \mathbb{Q}}\right)=0$ follows from an exact sequence:

$$
0 \rightarrow W I_{E, \mathbb{Q}} \rightarrow W \mathcal{O}_{Y, \mathbb{Q}} \rightarrow W \mathcal{O}_{E, \mathbb{Q}} \rightarrow 0 \text {. }
$$

\section{Rational chain connectedness}

In this section, we give a proof of the rational chain connectedness of threefolds of Fano type in characteristic $p>5$ even in the relative setting (cf. [Sho00, Theorem 1]).

Theorem 4.1. Let $k$ be a perfect field of characteristic $p>5$. Let $f: X \rightarrow$ $Y$ be a projective morphism with $f_{*} \mathcal{O}_{X}=\mathcal{O}_{Y}$ from a klt threefold $(X, \Delta)$ such that $-\left(K_{X}+\Delta\right)$ is $f$-nef and $f$-big. Then every fiber of $f$ is rationally chain connected.

First we recall the definition of varieties of Fano type and the rational chain connectedness.

Definition 4.2. Let $X$ be a projective normal variety. We say that $X$ is of Fano type if there exists an effective $\mathbb{Q}$-divisor $\Delta$ such that $-\left(K_{X}+\Delta\right)$ is ample and $(X, \Delta)$ is klt. 
Remark 4.3. If $\operatorname{dim} X \leq 3$ (cf. Remark 4.4) and there exists an effective $\mathbb{Q}$-divisor $\Delta$ such that $-\left(K_{X}+\Delta\right)$ is nef and big and $(X, \Delta)$ is klt, then $X$ is of Fano type (see [PS09, Lemma-Definition 2.6]) thanks to the existence of $\log$ resolutions.

Remark 4.4. For a klt pair $(X, B)$ and an effective $\mathbb{Q}$-Cartier $\mathbb{Q}$-divisor $D$, there exists $\epsilon>0$ such that $(X, B+\epsilon D)$ is again klt, if there exists a log resolution of $(X, B+D)$.

Definition 4.5 ([Kol96, IV. Definition 3.2]). A scheme $X$ of finite type over a field $k$ is called rationally chain connected if there exist a family of geometrically connected proper algebraic curves $g: U \rightarrow Y$ and a cycle morphism $u: U \rightarrow X$ with the following two conditions.

- The geometric fibers of $g$ have only rational components.

- $u^{(2)}: U \times_{Y} U \rightarrow X \times_{k} X$ is dominant.

Remark 4.6. We list some basic properties on the rational chain connectedness.

(1) ([Kol96, IV. Exercise 3.2.5] and [Tana, Proposition A.4]) For an arbitrary field extension $k^{\prime} / k, X$ is rationally chain connected if and only if so is the base change $X \times_{k} k^{\prime}$.

(2) ([Kol96, IV. Proposition 3.6]) Suppose that $k$ is an uncountable algebraically closed field. Then, $X$ is rationally chain connected if and only if a pair of general closed points can be connected by a chain of rational curves.

4.1. Birational and relative cases. In this subsection, we prove Theorem 4.1 when $\operatorname{dim} Y>0$. We start with a basic criterion for the rational chain connectedness.

Lemma 4.7. Let $f: X \rightarrow Y$ be a birational morphism of proper varieties over an algebraically closed field. Suppose that the following two conditions hold.

(1) Every fiber of $f$ is rationally chain connected.

(2) For a rational curve $C$ contained in $f(\operatorname{Ex}(f))$, its inverse image $f^{-1}(C)$ is rationally chain connected.

If a closed subscheme $\Gamma$ of $Y$ is rationally chain connected, then so is the inverse image $f^{-1}(\Gamma)$.

Proof. We can assume that $k$ is uncountable (Remark 4.6 (1)). First, we reduce the problem to the case where $\Gamma$ is a rational curve. Let $x_{1}, x_{2} \in$ $f^{-1}(\Gamma)$. If $f\left(x_{1}\right)=f\left(x_{2}\right)$, then $x_{1}$ and $x_{2}$ can be connected by a chain of rational curves in $f^{-1}(\Gamma)$ by the condition (1). Suppose $f\left(x_{1}\right) \neq f\left(x_{2}\right)$. By the rationally chain connectedness of $\Gamma$, there exists a connected chain $\Gamma^{\prime} \subset \Gamma$ of rational curves which contains $f\left(x_{1}\right)$ and $f\left(x_{2}\right)$. Since the existence of a connected chain of rational curves which contains $x_{1}$ and $x_{2}$ follows from the assertion for $\Gamma^{\prime}$, we may assume that $\Gamma$ is a connected chain of rational curves from the beginning. Moreover, by replacing $\Gamma$ with its irreducible component, we may assume that $\Gamma$ is a rational curve.

Suppose that $\Gamma$ is a rational curve. If $\Gamma \subset f(\operatorname{Ex}(f))$, then the assertion follows from the condition (2). Suppose that $\Gamma \not \subset f(\operatorname{Ex}(f))$. Set $\Gamma_{X}$ to 
be the proper transform of $\Gamma$ on $X$. Let $\Gamma \cap f(\operatorname{Ex}(f))=\left\{y_{1}, \cdots, y_{r}\right\}$. Since $f^{-1}(\Gamma)=\Gamma_{X} \cup \bigcup_{1 \leq i \leq r} f^{-1}\left(y_{i}\right)$, it is rationally chain connected by the condition (1).

We show the birational case (cf. [Sho00, Corollary 3]).

Theorem 4.8. Let $f: X \rightarrow Y$ be a proper birational morphism of normal threefolds over a perfect field of characteristic $p>5$. Suppose that $\left(Y, \Delta_{Y}\right)$ is klt for some effective $\mathbb{Q}$-divisor $\Delta_{Y}$. If a closed subscheme $\Gamma$ of $Y$ is rationally chain connected, then so is its inverse image $f^{-1}(\Gamma)$.

Proof. We may assume that the base field is an uncountable algebraically closed field.

First, we shall reduce to the case where $Y$ is $\mathbb{Q}$-factorial. Let $y_{1}, \cdots, y_{r}$ be the non- $\mathbb{Q}$-factorial points on $Y$. By Proposition 2.15 , we have a birational modification $g_{Y}: Y^{\prime} \rightarrow Y$ such that $Y^{\prime}$ is $\mathbb{Q}$-factorial and klt, $\operatorname{Ex}\left(g_{Y}\right)=$ $\coprod_{1 \leq i<r} g_{Y}^{-1}\left(y_{i}\right)$, and each $T_{i}=g_{Y}^{-1}\left(y_{i}\right)_{\text {red }}$ is a surface of Fano type. In particular, $T_{i}$ is rational, hence is rationally connected. Therefore, $g_{Y}^{-1}(\Gamma)$ is rationally chain connected. Further, there exist a normal threefold $X^{\prime}$ and birational morphisms $f^{\prime}: X^{\prime} \rightarrow Y^{\prime}$ and $X^{\prime} \rightarrow X$ such that the following diagram commutes:

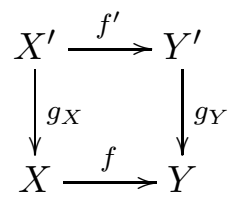

If we could prove that $f^{-1}\left(g_{Y}^{-1}(\Gamma)\right)$ is rationally chain connected, then so is its image $f^{-1}(\Gamma)=g_{X}\left(f^{\prime-1}\left(g_{Y}^{-1}(\Gamma)\right)\right)$ under $g_{X}$. Therefore, it is sufficient to prove the assertion for $f^{\prime}$. Hence, by replacing $Y$ with $Y^{\prime}$, we may assume that $Y$ is $\mathbb{Q}$-factorial.

Suppose $Y$ is $\mathbb{Q}$-factorial. We may assume $\Delta_{Y}=0$. Further, we may replace $f$ with a log resolution of $Y$. We run a $\left(K_{X}+E\right)$-MMP over $Y$, where $E$ is the reduced divisor with $\operatorname{Supp} E=\operatorname{Ex}(f)$. This MMP terminates and ends with $Y$ itself (see the proof of Theorem 3.14). Each step of this MMP is a pl-divisorial contraction or a pl-flip. Thus, it suffices to show the assertion under the assumption that $f: X \rightarrow Y$ is either a pl-divisorial contraction or pl-flipping contraction with respect to a dlt pair $(X, \Delta)$. The assertion follows from Lemma 4.7 and the argument in the proof in $\mathrm{GLP}^{+} 15$, Proposition 3.6].

Using the previous proposition, we prove Theorem 4.1 for $\operatorname{dim} Y>0$.

Proposition 4.9. Let $f: X \rightarrow Y$ be a projective morphism of normal varieties over a perfect field of characteristic $p>5$ with $f_{*} \mathcal{O}_{X}=\mathcal{O}_{Y}$. Suppose that $\operatorname{dim} X \leq 3$ and that there exists an effective $\mathbb{Q}$-divisor $\Delta$ on $X$ such that $(X, \Delta)$ is klt and $-\left(K_{X}+\Delta\right)$ is $f$-nef and $f$-big. If $\operatorname{dim} Y>0$, then every fiber of $f$ is rationally chain connected.

Proof. We may assume that the base field is an uncountable algebraically closed field. Fix a closed point $z \in Y$. We shall show that $f^{-1}(z)$ is rationally 
chain connected. By Proposition 2.15, there exists a commutative diagram

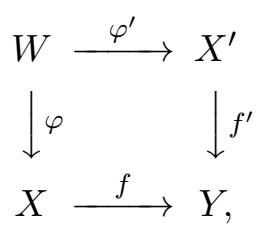

such that $\varphi$ and $\varphi^{\prime}$ are projective birational morphisms, and $f^{\prime-1}(z)$ is rationally connected. By Theorem 4.8, $\varphi^{\prime-1}\left(f^{\prime-1}(z)\right)$ is rationally chain connected, hence so is its image $f^{-1}(z)=\varphi\left(\varphi^{\prime-1}\left(f^{\prime-1}(z)\right)\right)$ under $\varphi$.

Remark 4.10. If $f: X \rightarrow Y$ is a Mori fiber space, then it is known that a general fiber of $f$ is rationally chain connected by [Tana, Theorem 0.4] in arbitrary positive characteristic.

4.2. Global case. In this subsection, we show the rational chain connectedness of log Fano threefolds.

Proposition 4.11. Let $X$ be a projective geometrically connected threefold of Fano type over a perfect field of characteristic $p>5$. Then $X$ is rationally chain connected.

Proof. We may assume that the base field is an uncountable algebraically closed field. Taking a small $\mathbb{Q}$-factorialization ([Bir, Theorem 1.6]) if necessary, we may assume that $X$ is $\mathbb{Q}$-factorial (cf. Remark 4.3). Let $\Delta$ be an effective $\mathbb{Q}$-divisor $\Delta$ such that $-\left(K_{X}+\Delta\right)$ is ample and $(X, \Delta)$ is klt. Then for any divisor $B$, we can run a $B$-MMP. Indeed, we can take an ample $\mathbb{Q}$-divisor $A$ and positive integer $m$ such that $\frac{1}{m} B \sim_{\mathbb{Q}} K_{X}+\Delta+A$ and $(X, \Delta+A)$ is klt. Then a $\left(K_{X}+\Delta+A\right)$-MMP with scaling of some ample divisor runs and terminates by $[\mathrm{BW}$, Theorem 1.6]. This MMP is also a $B$-MMP.

Let $f: X \rightarrow S$ be a maximally rationally chain connected fibration as in [Kol96, IV. Definition 5.1], where $S$ is a projective normal variety. This is almost holomorphic, i.e. we can find non-empty open subsets $X^{\prime} \subset X$ and $S^{\prime} \subset S$ such that $f$ induces a proper morphism $X^{\prime} \rightarrow S^{\prime}$. Note that $\operatorname{dim} S<\operatorname{dim} X$ by $\left[\mathrm{GLP}^{+} 15\right.$, Proposition 3.4 (1)]. Let $H$ be an ample Cartier divisor on $S$ and let $D$ be the closure of $\left.f^{*} H\right|_{X^{\prime}}$. We consider $(-D)$ MMP:

$$
X=: X_{0} \stackrel{\varphi_{0}}{\rightarrow} X_{1} \stackrel{\varphi_{1}}{\rightarrow} \cdots \stackrel{\varphi_{r-1}}{\rightarrow} X_{r} \rightarrow \cdots
$$

Note that this MMP ends with a Mori fiber space. Let $D_{i}$ be the strict transform of $D$ on $X_{i}$. Let $r$ be the least $r$ satisfying the following condition:

- for the $\left(-D_{r}\right)$-negative extremal contraction $\psi_{r}: X_{r} \rightarrow W_{r}$ yielding $\varphi_{r}$, its exceptional locus $\operatorname{Ex}\left(\psi_{r}\right)$ is intersecting with a general fiber of $f_{r}: X_{r} \rightarrow S$, i.e. $\operatorname{Ex}\left(\psi_{r}\right)$ dominates $S$.

Note that the induced rational map $f_{i}: X_{i} \rightarrow S$ is also a maximally rationally chain connected fibration for any $0 \leq i \leq r$ and when $\psi_{i}$ is a Mori fiber space, we set $\operatorname{Ex}\left(\psi_{i}\right):=X_{i}$ (hence such $r$ always exists).

Suppose that $\operatorname{dim} S>0$ by contradiction. Fix very general $s \in S$. Then $s$ satisfies the following property (see [Kol96, IV. (5.2.1)]): if $f_{r}^{-1}(s)$ intersects with a rational curve $C \subset X_{r}$ then $C \subset f_{r}^{-1}(s)$. By Proposition 4.9 for 
$\operatorname{dim} W_{r}>0$ and $\mathrm{GLP}^{+} 15$, Proposition $\left.3.4(2)\right]$ for $\operatorname{dim} W_{r}=0$, some $\psi_{r^{-}}$ contracted rational curve $C$ intersects with $f_{r}^{-1}(s)$, and hence $C \subset f_{r}^{-1}(s)$. Since $D_{r}$ is the pull-back of $H$ by $f_{r}$ around $C$, it follows that $C \cdot D_{r}=0$. However, this contradicts the $\left(-D_{r}\right)$-negativity of $\psi_{r}$.

Remark 4.12. By the same proof as above, Proposition 4.11 can be generalized to arbitrary dimension if we assume the existence of log resolution and the minimal model program.

Proof of Theorem 4.1. We may assume that $-\left(K_{X}+\Delta\right)$ is $f$-ample. When $\operatorname{dim} Y>0$, the assertion follows from Proposition 4.9, When $\operatorname{dim} Y=0$, the assertion follows from Proposition 4.11.

\section{RATIONAL POINTS ON LOG FANO CONTRACTIONS OVER FINITE FIELDS}

In this section, we discuss rational points on a (mainly Fano) variety over a finite field.

First, we treat the birational case as follows:

Theorem 5.1. Let $f: X \rightarrow Y$ be a birational morphism of normal threefolds over $\mathbb{F}_{q}$ of characteristics $p>5$. If there exist effective $\mathbb{Q}$-divisors $\Delta_{X}$ on $X$ and $\Delta_{Y}$ on $Y$ such that $\left(X, \Delta_{X}\right)$ and $\left(Y, \Delta_{Y}\right)$ are klt. Then

$$
\# X\left(\mathbb{F}_{q}\right) \equiv \# Y\left(\mathbb{F}_{q}\right) \quad(\bmod q) .
$$

Proof. Taking a resolution, we may assume that $X$ is smooth and that $f$ is a morphism. Since $Y$ has $W \mathcal{O}$-rational singularities by Theorem 3.16, the assertion holds by Remark 3.1 and [BBE07, Proposition 6.9].

We also have a global statement as follows.

Corollary 5.2. Let $X \rightarrow Y$ be a birational map of proper klt threefolds over $\mathbb{F}_{q}$ of characteristics $p>5$. Then $\# X\left(\mathbb{F}_{q}\right) \equiv \# Y\left(\mathbb{F}_{q}\right)(\bmod q)$.

Proof. Taking a common resolution, the assertion follows from Theorem 5.1 .

Now, we prove one of the main theorems of this paper.

Theorem 5.3. Let $X$ be a proper geometrically connected threefold over a finite field $\mathbb{F}_{q}$ of characteristic $p>5$. Suppose that $(X, \Delta)$ is klt for some effective $\mathbb{Q}$-divisor $\Delta$ and $X$ is rationally chain connected.

Then $\# X\left(\mathbb{F}_{q}\right) \equiv 1(\bmod q)$ holds. In particular, $X$ has a rational point.

Proof. Taking a resolution of singularities, we may assume that $X$ is smooth by Theorem 4.8 and Theorem 5.1. Then the assertion follows from Esn03, Theorem 1.1] and the argument between (1.1) and (1.4) in Esn03.

Next, we consider the relative case.

Theorem 5.4. Let $f: X \rightarrow Y$ be a proper morphism of normal varieties over $\mathbb{F}_{q}$ of characteristics $p>5$ with $f_{*} \mathcal{O}_{X}=\mathcal{O}_{Y}$. Suppose that there exists an effective $\mathbb{Q}$-divisor $\Delta$ such that $(X, \Delta)$ is a klt threefold and $-\left(K_{X}+\Delta\right)$ is $f$-nef and $f$-big. Then

$$
\# X_{y}\left(\mathbb{F}_{q}\right) \equiv 1 \quad(\bmod q)
$$


holds for any $\mathbb{F}_{q}$-rational point $y$ on $Y$ and the fiber $X_{y}$ over $y$. In particular,

$$
\# X\left(\mathbb{F}_{q}\right) \equiv \# Y\left(\mathbb{F}_{q}\right) \quad(\bmod q) .
$$

Proof. We shall prove the first assertion, since the second assertion follows from the first assertion. We may assume that $\operatorname{dim} Y>0$ by Theorem 5.3 . Further, we may assume that $f$ is projective by Chow's lemma and Theorem 5.1. Since the statement is local on $Y$, we may also assume that $Y$ has a unique $\mathbb{F}_{q}$-rational point $y$.

By Proposition 2.15, we have a commutative diagram

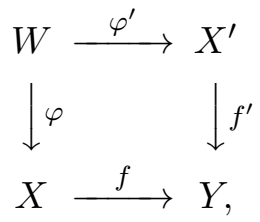

such that $\varphi$ and $\varphi^{\prime}$ are projective birational morphisms, and $S:=f^{\prime-1}(y)$ is a surface of Fano type. By Theorem 5.1, we have

$$
\# X\left(\mathbb{F}_{q}\right) \equiv \# X^{\prime}\left(\mathbb{F}_{q}\right) \quad(\bmod q)
$$

Further $\# X^{\prime}\left(\mathbb{F}_{q}\right)=\# S\left(\mathbb{F}_{q}\right)$ holds as $y$ is the unique rational point on $Y$. Since $S$ is a surface of Fano type (hence a rational surface with rational singularities) it follows that $\# S\left(\mathbb{F}_{q}\right) \equiv 1(\bmod q)$, which shows

$$
\# X_{y}\left(\mathbb{F}_{q}\right)=\# X\left(\mathbb{F}_{q}\right) \equiv \# X^{\prime}\left(\mathbb{F}_{q}\right)=\# S\left(\mathbb{F}_{q}\right) \equiv 1 \quad(\bmod q) \text {. }
$$

By the same technique as above, we can also show the following vanishing theorem.

Theorem 5.5. Let $X$ be a proper threefold over a perfect field of characteristic $p>5$. Suppose that $(X, \Delta)$ is klt for some effective $\mathbb{Q}$-divisor $\Delta$ and $X$ is rationally chain connected. Then $H^{i}\left(X, W \mathcal{O}_{X, \mathbb{Q}}\right)=0$ for $i>0$.

Proof. Let $\varphi: V \rightarrow X$ be a birational morphism from a smooth projective threefold $V$. Since $V$ is also rationally chain connected (Theorem 4.8), we obtain $H^{i}\left(V, W \mathcal{O}_{V}\right) \otimes_{\mathbb{Z}} \mathbb{Q}=0$ for $i>0$ by Esn03, Theorem 1.1]. By Remark 2.17, we have that $H^{i}\left(V, W \mathcal{O}_{V, \mathbb{Q}}\right)=0$ for $i>0$. Since $X$ has $W \mathcal{O}_{\text {- }}$ rational singularities, it follows that $R^{i} \varphi_{*}\left(W \mathcal{O}_{V, \mathbb{Q}}\right)=0$ for $i>0$, which proves $H^{i}\left(X, W \mathcal{O}_{X, \mathbb{Q}}\right)=0$ for $i>0$ by the Leray spectral sequence.

Lastly, we conclude the main theorems using Theorem 4.1 .

Proof of Theorem 1.2. This follow from Theorem 4.1 and Theorem 5.3 .

Proof of Theorem 1.3. This follow from Theorem 4.1 and Theorem 5.5.

\section{REFERENCES}

[Abh66] S. S. Abhyankar, Resolution of singularities of embedded algebraic surfaces., Pure and Applied Mathematics, vol. 24, Academic Press, New York-London, 1966.

[BBE07] P. Berthelot, S. Bloch, and H. Esnault, On Witt vector cohomology for singular varieties, Compos. Math. 143 (2007), no. 2, 363-392.

[Bir12] C. Birkar, Existence of log canonical flips and a special LMMP, Publ. Math. Inst. Hautes Études Sci. (2012), 325-368. 
[Bir] _ Existence of flips and minimal models for 3-folds in char p, to appear in Ann. Sci. Ecole Norm. Sup., available at arXiv:1311.3098v2.

[BW] C. Birkar and J. Waldron, Existence of Mori fibre spaces for 3-folds in char $p$, available at arXiv:1410.4511v1.

[BE08] M. Blickle and H. Esnault, Rational singularities and rational points, Pure Appl. Math. Q. 4 (2008), no. 3, 729-741.

[Cam92] F. Campana, Connexité rationnelle des variétés de Fano, Ann. Sci. École Norm. Sup. (4) 25 (1992), no. 5, 539-545 (French).

[CMM14] P. Cascini, J. McKernan, and M. Mustaţă, The augmented base locus in positive characteristic, Proc. Edinb. Math. Soc. (2) 57 (2014), no. 1, 79-87.

[CR11] A. Chatzistamatiou and K. Rülling, Higher direct images of the structure sheaf in positive characteristic, Algebra Number Theory 5 (2011), no. 6, 693-775.

[CR12] _ Hodge-Witt cohomology and Witt-rational singularities, Doc. Math. 17 (2012), 663-781.

[CP08] V. Cossart and O. Piltant, Resolution of singularities of threefolds in positive characteristic. I. Reduction to local uniformization on Artin-Schreier and purely inseparable coverings, J. Algebra 320 (2008), no. 3, 1051-1082.

[dJ97] A. J. de Jong, Families of curves and alterations, Ann. Inst. Fourier (Grenoble) 47 (1997), no. 2, 599-621.

[Esn03] H. Esnault, Varieties over a finite field with trivial Chow group of 0-cycles have a rational point, Invent. Math. 151 (2003), no. 1, 187-191.

[Fuj07] O. Fujino, Special termination and reduction to pl flips, Flips for 3-folds and 4-folds, Oxford Lecture Ser. Math. Appl., vol. 35, Oxford Univ. Press, Oxford, 2007, pp. 63-75.

[FT12] O. Fujino and H. Tanaka, On log surfaces, Proc. Japan Acad. Ser. A Math. Sci. 88 (2012), no. 8, 109-114.

$\left[\right.$ GLP $\left.^{+} 15\right]$ Y. Gongyo, Z. Li, Z. Patakfalvi, K. Schwede, H. Tanaka, and R. Zong, On rational connectedness of globally F-regular threefolds, Adv. Math. 280 (2015), $47-78$.

[Gro65] A. Grothendieck, Éléments de géométrie algébrique. IV. Étude locale des schémas et des morphismes de schémas. II, Inst. Hautes Études Sci. Publ. Math. 24 (1965), 231 (French).

[HM07] C. D. Hacon and J. Mckernan, On Shokurov's rational connectedness conjecture, Duke Math. J. 138 (2007), no. 1, 119-136.

[HX15] C. D. Hacon and C. Xu, On the three dimensional minimal model program in positive characteristic, J. Amer. Math. Soc. 28 (2015), no. 3, 711-744.

[Ill79] L. Illusie, Complexe de de Rham-Witt et cohomologie cristalline, Ann. Sci. École Norm. Sup. (4) 12 (1979), no. 4, 501-661 (French).

[KMM87] Y. Kawamata, K. Matsuda, and K. Matsuki, Introduction to the minimal model problem, Algebraic geometry, Sendai, 1985, Adv. Stud. Pure Math., vol. 10, North-Holland, Amsterdam, 1987, pp. 283-360.

[Kee99] S. Keel, Basepoint freeness for nef and big line bundles in positive characteristic, Ann. of Math. (2) 149 (1999), no. 1, 253-286.

[KEW] M. Kerz, H. Esnault, and O. Wittenberg, A restriction isomorphism for cycles of relative dimension zero, available at arXiv:1503.08187v1

[Kol96] J. Kollár, Rational curves on algebraic varieties, Ergebnisse der Mathematik und ihrer Grenzgebiete. 3. Folge. A Series of Modern Surveys in Mathematics, vol. 32, Springer-Verlag, Berlin, 1996.

[Kol97] _ Quotient spaces modulo algebraic groups, Ann. of Math. (2) 145 (1997), no. $1,33-79$.

[Kol13] _ Singularities of the minimal model program, Cambridge Tracts in Mathematics, vol. 200, Cambridge University Press, Cambridge, 2013. With a collaboration of Sándor Kovács.

[KMM92] J. Kollár, Y. Miyaoka, and S. Mori, Rational connectedness and boundedness of Fano manifolds, J. Differential Geom. 36 (1992), no. 3, 765-779.

[KM98] J. Kollár and S. Mori, Birational geometry of algebraic varieties, Cambridge Tracts in Mathematics, vol. 134, Cambridge University Press, Cambridge, 1998. 
[Mad] Z. Maddock, Regular del Pezzo surfaces with irregularity, available at arXiv: $1304.5555 \mathrm{v} 1$

[Poo04] B. Poonen, Bertini theorems over finite fields, Ann. of Math. 160 (2004), no. 3, 1099-1127.

[PS09] Yu. G. Prokhorov and V. V. Shokurov, Towards the second main theorem on complements, J. Algebraic Geom. 18 (2009), no. 1, 151-199.

[Sch07] S. Schröer, Weak del Pezzo surfaces with irregularity, Tohoku Math. J. (2) 59 (2007), no. 2, 293-322.

[SS10] K. Schwede and K. E. Smith, Globally F-regular and log Fano varieties, Adv. Math. 224 (2010), no. 3, 863-894.

[Sho00] V. V. Shokurov, On rational connectedness, Mat. Zametki 68 (2000), no. 5, 771-782 (Russian, with Russian summary); English transl., Math. Notes 68 (2000), no. 5-6, 652-660.

[Ser79] J.-P. Serre, Local fields, Graduate Texts in Mathematics, vol. 67, SpringerVerlag, New York-Berlin, 1979. Translated from the French by Marvin Jay Greenberg.

[Tan14] H. Tanaka, Minimal models and abundance for positive characteristic log surfaces, Nagoya Math. J. 216 (2014), 1-70.

[Tan15] — The X-method for klt surfaces in positive characteristic, J. Algebraic Geom. 24 (2015), no. 4, 605-628.

[Tan16] Abundance theorem for semi log canonical surfaces in positive characteristic, Osaka J. Math. 53 (2016), no. 2, 535-566.

[Tana] _ Behavior of canonical divisors under purely inseparable base changes, to appear in J. Reine und Angew. Math., available at arXiv:1502.01381v3.

[Tanb] _ Semiample perturbations for log canonical varieties over an F-finite field containing an infinite perfect field, available at arXiv:1503.01264v3.

[Wan] Y. Wang, On relative rational chain connectedness of threefolds with anti-big canonical divisors in positive characteristics, available at $\operatorname{arXiv:1512.06189\mathrm {v}1\text {.}}$

[Xu14] C. Xu, Finiteness of algebraic fundamental groups, Compos. Math. 150 (2014), no. 3, 409-414.

[Xu15] _ On the base-point-free theorem of 3-folds in positive characteristic, J. Inst. Math. Jussieu 14 (2015), no. 3, 577-588.

[Zha06] Q. Zhang, Rational connectedness of log Q-Fano varieties, J. Reine Angew. Math. 590 (2006), 131-142.

Graduate School of Mathematical Sciences, the University of Tokyo, 3-8-1 Komaba, Meguro-Ku, TOKyo 153-8914, Japan.

E-mail address: gongyo@ms.u-tokyo.ac.jp

Graduate School of Mathematical Sciences, the University of Tokyo, 3-8-1 Komaba, Meguro-Ku, Tokyo 153-8914, Japan.

E-mail address: nakamura@ms.u-tokyo.ac.jp

Department of Mathematics, Imperial College, London, 180 Queen's Gate, LONDON SW7 2AZ, UK

E-mail address: h.tanaka@imperial.ac.uk 